QUARTERLY OF APPLIED MATHEMATICS

VOLUME LXV, NUMBER 2

JUNE 2007, PAGES 317-337

$\mathrm{S} 0033-569 \mathrm{X}(07) 01056-6$

Article electronically published on March 7, 2007

\title{
THE EXISTENCE OF EIGHT POSITIVE PERIODIC SOLUTIONS FOR A GENERALIZED PREY-PREDATOR SYSTEM WITH DELAY AND STOCKING
}

\author{
BY \\ ZHENGQIU ZHANG (School of Mathematics and Computing Technology, Central South University, \\ Changsha, 410083, People's Republic of China) \\ AND \\ XINSHENG XIONG (Department of Applied Mathematics, Hunan University, Changsha, 410082, \\ People's Republic of China)
}

\begin{abstract}
By means of using Mawhin's continuation theorem of coincidence degree theory, we derive criteria for the existence of eight positive periodic solutions of a generalized prey-predator system with time delay and stocking.
\end{abstract}

1. Introduction. Freedman and Waltman 44 considered a general model of two predators competing for a single prey. They derived criteria for strong persistence in terms of conditions on the system parameters. Farkas and Freedman [2] obtained criteria for global stability of a general model of a two-predator-one-prey system in terms of restrictions on the growth functions. Motivated by the work of Freedman and Ruan [3] for retarded functional differential equations, Mukherjee and and Roy [7] investigated a model representing a resource (prey) and two predators with a delay due to gestation and derived conditions for uniform persistence. The existence conditions for a positive equilibrium were discussed. Since global stability is of great interest, they provided sufficient conditions in terms of the parameters of the system to guarantee it. In [8, motivated by the work of Mukherjee and Roy [7] for constructing a generalized model of a two-predator-one-prey system, we constructed a mathematical model, representing a

Received February 3, 2006.

2000 Mathematics Subject Classification. Primary 34K13, 34K60.

Key words and phrases. Eight positive periodic solutions, a generalized prey-predator system, delay and stocking, the continuation theorem of coincidence degree theory, topological degree theory.

This project was supported by NNSF of China (10271044) and by Chinese postdoctoral science fund (20060400267).

E-mail address: z_q_zhang@sina.com.cn

E-mail address: xxs82@163.com

(C)2007 Brown University 
food chain system with delay due to gestation:

$$
\left\{\begin{array}{l}
\frac{d x_{1}}{d t}=x_{1} g_{1}\left(t, x_{1}\right)-c_{1}(t) x_{2} p_{2}\left(x_{1}\right) \\
\frac{d x_{2}}{d t}=x_{2} g_{2}\left(t, x_{2}\right)+\beta_{1}(t) x_{2} p_{2}\left(x_{1}(t-\tau)\right)-\beta_{2}(t) x_{3} p_{3}\left(x_{2}\right), \\
\frac{d x_{3}}{d t}=x_{3}\left[-d_{1}(t)+\beta_{3}(t) p_{3}\left(x_{2}(t-\tau)\right)-a_{31}(t) x_{3}\right] .
\end{array}\right.
$$

In $[8$, by using the continuation theorem of coincidence degree theory, we studied the existence of positive periodic solutions of system (1.1). In this paper, we consider the following food chain system with stocking and delay:

$$
\left\{\begin{array}{l}
\frac{d x_{1}}{d t}=x_{1} g_{1}\left(t, x_{1}\right)-c_{1}(t) x_{2} p_{2}\left(x_{1}\right)-h_{1}(t), \\
\frac{d x_{2}}{d t}=-x_{2} g_{2}\left(t, x_{2}\right)+\beta_{1}(t) x_{2} p_{2}\left(x_{1}(t-\tau)\right)-\beta_{2}(t) x_{3} p_{3}\left(x_{2}\right)-h_{2}(t), \\
\frac{d x_{3}}{d t}=x_{3}\left[-d_{1}(t)+\beta_{3}(t) p_{3}\left(x_{2}(t-\tau)\right)-a_{31}(t) x_{3}(t)\right]-h_{3}(t),
\end{array}\right.
$$

where $\tau>0$ is a constant time delay. In system (1.2), all parameters are positive continuous $\omega$-periodic functions with period $\omega>0 ; x_{1}(t)$ denotes the biomass at time $t$ of resource species $x_{1}, x_{2}(t)$ and $x_{3}(t)$ represent the densities of the species $x_{2}$ and $x_{3}$, respectively, $\beta_{1}$ and $\beta_{3}$ are the conversion of biomass constants, respectively, and $d_{1}$ is the death rate of species $x_{3}, h_{1}, h_{2}, h_{3}$ are the stocking of species $x_{1}$, species $x_{2}$ and species $x_{3}$, respectively. For biological relevance of system (1.2), see [1.

The following assumptions on the functions $g_{i}(t, x), i=1,2, p_{i}(x), i=2,3$, are given below. (H1): $g_{i}(t, x)$ are continuous and $\omega$-periodic with respect to $t, i=1,2, g_{1}(t, 0)>0$, $g_{1}(t, x)$ is monotonely decreasing in $x$ for fixed $t, t, x \in R, g_{2}(t, x)$ is monotonely increasing in $x$ for fixed $t, g_{2}(t, 0)>0, g_{2}(t, x)>0$, for $x>0, t, x \in R$. (H2) $p_{i}(x)$ are monotonely increasing and $p_{i}(x)>0$, for $x>0, i=2,3$.

On the existence of periodic solutions for system (1.2), so far, few results have been found in the literature. This motivates us to consider the study of the existence for periodic solutions of system (1.2). Since the existence results on multiple periodic solutions of delayed population models obtained by means of coincidence degree theory are very scarce, then in this paper, instead of investigating the existence of a periodic solution, we investigate the existence of multiple positive periodic solutions for system (1.2) by using the continuation theorem of coincidence degree theory. The paper is organized as follows. In Section 2, by applying the continuation theorem of coincidence degree theory, we establish a sufficient condition for the existence of eight positive periodic solutions of system (1.2). In Section 3, we illustrate our result with two examples.

2. Main result. For the reader's convenience, we first summarize a few concepts from the book by Gaines and Mawhin [5, 6].

Let $X$ and $Z$ be normed vector spaces. Let $L$ : Dom $L \subset X \rightarrow Z$ be a linear mapping and $N: X \rightarrow Z$ be a continuous mapping. The mapping $L$ will be called a Fredholm mapping of index zero if $\operatorname{dim} \operatorname{Ker} L=\operatorname{codim} \operatorname{Im} L<\infty$ and $\operatorname{Im} L$ is closed in $Z$. If $L$ is a Fredholm mapping of index zero, then there exist continuous projectors $P: X \rightarrow X$ and $Q: Z \rightarrow Z$ such that $\operatorname{ImP}=\operatorname{Ker} L$ and $\operatorname{Im} L=\operatorname{Ker} Q=\operatorname{Im}(I-Q)$. It follows that $\left.L\right|_{\text {DomL LKerP }}:(I-P) X \rightarrow \operatorname{ImL}$ is invertible and its inverse is denoted by $K_{p}$. If $\Omega$ is a bounded open subset of $X$, the mapping $N$ is called $L$-compact on $\bar{\Omega}$ if $(Q N)(\bar{\Omega})$ is 
bounded and $K_{p}(I-Q) N: \bar{\Omega} \rightarrow X$ is compact. Because $\operatorname{Im} Q$ is isomorphic to Ker $L$, there exists an isomorphism $J: \operatorname{Im} Q \rightarrow \operatorname{Ker} L$.

In the proof of our existence result, we need the following continuation theorem.

Theorem 2.1 (Continuation Theorem, Gaines and Mawhin [5, 6]). Let $L$ be a Fredholm mapping of index zero and let $N$ be $L$-compact on $\bar{\Omega}$. Suppose

(a) for each $\lambda \in(0,1)$, every solution $x$ of $L x=\lambda N x$ is such that $x \notin \partial \Omega$;

(b) $Q N x \neq 0$ for each $x \in \partial \Omega \cap \operatorname{Ker} L$;

(c) $\operatorname{deg}\{J Q N, \Omega \cap$ Ker $L, 0\} \neq 0$.

Then the equation $L x=N x$ has at least one solution lying in Dom $L \cap \bar{\Omega}$.

For the sake of convenience and simplicity, we introduce some notation as follows:

$$
\max _{t \in[0, \omega]} g_{1}(t, 0)=a_{1}^{M}, \quad f^{M}=\max _{t \in[0, \omega]} f(t), \quad f^{l}=\min _{t \in[0, \omega]} f(t) ;
$$

here $f$ is a positive continuous $\omega$-periodic function.

Theorem 2.2. Assume that the following conditions hold:

(i) There exists a constant $A$ such that when $x \geq A$,

$$
g_{1}\left(t, e^{x}\right) \leq 0, \quad \forall t, x \in R .
$$

(ii) There exist a constant $B$ such that when $x \geq B$,

$$
g_{2}\left(t, e^{x}\right) \geq \beta_{1}^{M} p_{2}\left(e^{A}\right), \quad \forall t, x \in R .
$$

(iii) There exist two positive constants $l_{ \pm}$with $\frac{h_{1}^{l}}{a_{1}^{M}}<l_{-}<l_{+}<A$ such that $\forall t, x \in R$,

$$
e^{x} g_{1}\left(t, e^{x}\right) \geq c_{1}^{M} e^{B} p_{2}\left(e^{x}\right)+h_{1}^{M}, \quad \ln l_{-} \leq x \leq \ln l_{+} .
$$

(iv) There exist two positive constants $u_{ \pm}$with $\frac{h_{2}^{l}}{\beta_{1}^{M} p_{2}\left(e^{A}\right)}<u_{-}<u_{+}<B$ such that $\forall t, x \in R$;

$$
h_{2}^{M}+\frac{\beta_{2}^{M} \beta_{3}^{M}}{a_{31}^{l}} p_{3}\left(e^{B}\right) p_{3}\left(e^{x}\right)+e^{x} g_{2}\left(t, e^{x}\right) \leq \beta_{1}^{l} e^{x} p_{2}\left(\frac{h_{1}^{l}}{a_{1}^{M}}\right), \ln u_{-} \leq x \leq \ln u_{+} .
$$

(v) $\beta_{3}^{l} p_{3}\left[\frac{h_{2}^{l}}{\beta_{1}^{M} p_{2}\left(e^{A}\right)}\right]>d_{1}^{M}+2 \sqrt{a_{31}^{M} h_{3}^{M}}$.

Then system (1.2) has at least eight positive $\omega$-periodic solutions.

Proof. Since we are concerned with positive periodic solutions of system (1.2), we make the change of variables,

$$
x_{i}(t)=e^{u_{i}(t)}, \quad i=1,2,3 .
$$

Then system (1.2) is rewritten as

$$
\left\{\begin{array}{l}
\frac{d u_{1}(t)}{d t}=g_{1}\left(t, e^{u_{1}(t)}\right)-c_{1}(t) e^{u_{2}(t)-u_{1}(t)} p_{2}\left(e^{u_{1}(t)}\right)-h_{1}(t) e^{-u_{1}(t)} \\
\frac{d u_{2}(t)}{d t}=-g_{2}\left(t, e^{u_{2}(t)}\right)+\beta_{1}(t) p_{2}\left(e^{u_{1}(t-\tau)}\right)-\beta_{2}(t) e^{u_{3}(t)-u_{2}(t)} p_{3}\left(e^{u_{2}(t)}\right)-h_{2}(t) e^{-u_{2}(t)} \\
\frac{d u_{2}(t)}{d t}=-d_{1}(t)+\beta_{3}(t) p_{3}\left(e^{u_{2}(t-\tau)}\right)-a_{31}(t) e^{u_{3}(t)}-h_{3}(t) e^{-u_{3}(t)}
\end{array}\right.
$$


To prove the existence of positive periodic solutions for system (1.2), because of (2.1), we only need to show the existence of periodic solutions for system (2.2). To apply Theorem 2.1 to system (2.2), we take

$$
X=Z=\left\{u=\left(u_{1}, u_{2}, u_{3}\right)^{T} \in C\left(R, R^{3}\right): u(t+\omega)=u(t)\right\}
$$

and define

$$
\|u\|=\sum_{i=1}^{3} \max _{t \in[0, \omega]}\left|u_{i}(t)\right|, \quad u \in X \quad \text { or } \quad Z .
$$

Equipped with the above norm $\|\|,$.$X and Z$ are Banach spaces.

Let

$$
\begin{gathered}
N u=\left[\begin{array}{l}
g_{1}\left(t, e^{u_{1}(t)}\right)-c_{1}(t) e^{u_{2}(t)-u_{1}(t)} p_{2}\left(e^{u_{1}(t)}\right)-h_{1}(t) e^{-u_{1}(t)} \\
-g_{2}\left(t, e^{u_{2}(t)}\right)+\beta_{1}(t) p_{2}\left(e^{u_{1}(t-\tau)}\right)-\beta_{2}(t) e^{u_{3}(t)-u_{2}(t)} p_{3}\left(e^{u_{2}(t)}\right)-h_{2}(t) e^{-u_{2}(t)} \\
-d_{1}(t)+\beta_{3}(t) p_{3}\left(e^{u_{2}(t-\tau)}\right)-a_{31}(t) e^{u_{3}(t)}-h_{3}(t) e^{-u_{3}(t)}
\end{array}\right], u \in X, \\
L u=u^{\prime}=\frac{d u(t)}{d t}, \quad P u=\frac{1}{\omega} \int_{0}^{\omega} u(t) d t, \quad u \in X ; \quad Q z=\frac{1}{\omega} \int_{0}^{\omega} z(t) d t, \quad z \in Z .
\end{gathered}
$$

Then it follows that

$\operatorname{Ker} L=R^{3}, \operatorname{Im} L=\left\{z \in Z: \int_{0}^{\omega} z(t) d t=0\right\}$ is closed in $Z, \operatorname{dimKer} L=3=\operatorname{codim} \operatorname{Im} L$, and $P, Q$ are continuous projectors such that

$$
\operatorname{ImP}=\operatorname{Ker} L, \quad \operatorname{Ker} Q=\operatorname{Im} L=\operatorname{Im}(I-Q) .
$$

Therefore, $L$ is a Fredholm mapping of index zero. Furthermore, the generalized inverse (to $L) K_{p}: \operatorname{Im} L \rightarrow \operatorname{Ker} P \cap D o m L$ is given by

$$
K_{p}(z)=\int_{0}^{t} z(s) d s-\frac{1}{\omega} \int_{0}^{\omega} \int_{0}^{t} z(s) d s d t .
$$

Then

$$
Q N u=\left[\begin{array}{c}
\frac{1}{\omega} \int_{0}^{\omega} F_{1}(s) d s \\
\frac{1}{\omega} \int_{0}^{\omega} F_{2}(s) d s \\
\frac{1}{\omega} \int_{0}^{\omega} F_{3}(s) d s
\end{array}\right]
$$

and

$$
K_{p}(I-Q) N u=\left[\begin{array}{c}
\int_{0}^{t} F_{1}(s) d s-\frac{1}{\omega} \int_{0}^{\omega} \int_{0}^{t} F_{1}(s) d s d t+\left(\frac{1}{2}-\frac{t}{\omega}\right) \int_{0}^{\omega} F_{1}(s) d s \\
\int_{0}^{t} F_{2}(s) d s-\frac{1}{\omega} \int_{0}^{\omega} \int_{0}^{t} F_{2}(s) d s d t+\left(\frac{1}{2}-\frac{t}{\omega}\right) \int_{0}^{\omega} F_{2}(s) d s \\
\int_{0}^{t} F_{3}(s) d s-\frac{1}{\omega} \int_{0}^{\omega} \int_{0}^{t} F_{3}(s) d s d t+\left(\frac{1}{2}-\frac{t}{\omega}\right) \int_{0}^{\omega} F_{3}(s) d s
\end{array}\right]
$$

where

$$
\begin{gathered}
F_{1}(s)=g_{1}\left(s, e^{u_{1}(s)}\right)-c_{1}(s) e^{u_{2}(s)-u_{1}(s)} p_{2}\left(e^{u_{1}(s)}\right)-h_{1}(s) e^{-u_{1}(s)} \\
F_{2}(s)=-g_{2}\left(s, e^{u_{2}(s)}\right)+\beta_{1}(s) p_{2}\left(e^{u_{1}(s-\tau)}\right)-\beta_{2}(s) e^{u_{3}(s)-u_{2}(s)} p_{3}\left(e^{u_{2}(s)}\right)-h_{2}(s) e^{-u_{2}(s)}, \\
F_{3}(s)=-d_{1}(s)+\beta_{3}(s) p_{3}\left(e^{u_{2}(s-\tau)}\right)-a_{31}(s) e^{u_{3}(s)}-h_{3}(s) e^{-u_{3}(s)}
\end{gathered}
$$


Obviously, $Q N$ and $K_{p}(I-Q) N$ are continuous. It is not difficult to show that $K_{p}(I-Q) N(\bar{\Omega})$ is compact for any open bounded set $\Omega \subset X$ by using the ArzelaAscoli theorem. Moreover, $Q N(\bar{\Omega})$ is clearly bounded. Thus, $N$ is $L$-compact on $\bar{\Omega}$ with any open bounded set $\Omega \subset X$.

Corresponding to the operator equation $L x=\lambda N x, \lambda \in(0,1)$. we have

$$
\left\{\begin{aligned}
\frac{d u_{1}(t)}{d t}= & {\left[g_{1}\left(t, e^{u_{1}(t)}\right)-c_{1}(t) e^{u_{2}(t)-u_{1}(t)} p_{2}\left(e^{u_{1}(t)}\right)-h_{1}(t) e^{-u_{1}(t)}\right], } \\
\frac{d u_{2}(t)}{d t}= & {\left[-g_{2}\left(t, e^{u_{2}(s)}\right)+\beta_{1}(t) p_{2}\left(e^{u_{1}(t-\tau)}\right)\right.} \\
& \left.-\beta_{2}(t) e^{u_{3}(t)-u_{2}(t)} p_{3}\left(e^{u_{2}(t)}\right)-h_{2}(t) e^{-u_{2}(t)}\right] \\
\frac{d u_{3}(t)}{d t}= & {\left[-d_{1}(t)+\beta_{3}(t) p_{3}\left(e^{u_{2}(t-\tau)}\right)-a_{31}(t) e^{u_{3}(t)}-h_{3}(t) e^{-u_{3}(t)}\right] . }
\end{aligned}\right.
$$

Assume that $u=u(t) \in X$ is a solution of system (2.3) for a certain $\lambda \in(0,1)$. Since $\left(u_{1}, u_{2}, u_{3}\right)^{T} \in X$, there exist $\xi_{i}, \eta_{i} \in[0, \omega]$ such that

$$
u_{i}\left(\xi_{i}\right)=\max _{t \in[0, \omega]} u_{i}(t), \quad u_{i}\left(\eta_{i}\right)=\min _{t \in[0, \omega]} u_{i}(t), \quad i=1,2,3 .
$$

Then

$$
u_{i}^{\prime}\left(\eta_{i}\right)=0, \quad u_{i}^{\prime}\left(\xi_{i}\right)=0, \quad i=1,2,3 .
$$

From this and system (2.3), we obtain

$$
\left\{\begin{array}{l}
g_{1}\left(\xi_{1}, e^{u_{1}\left(\xi_{1}\right)}\right)-c_{1}\left(\xi_{1}\right) e^{u_{2}\left(\xi_{1}\right)-u_{1}\left(\xi_{1}\right)} p_{2}\left(e^{u_{1}\left(\xi_{1}\right)}\right)-h_{1}\left(\xi_{1}\right) e^{-u_{1}\left(\xi_{1}\right)}=0, \\
-g_{2}\left(\xi_{2}, e^{u_{2}\left(\xi_{2}\right)}\right)+\beta_{1}\left(\xi_{2}\right) p_{2}\left(e^{u_{1}\left(\xi_{2}-\tau\right)}\right)-\beta_{2}\left(\xi_{2}\right) e^{u_{3}\left(\xi_{2}\right)-u_{2}\left(\xi_{2}\right)} p_{3}\left(e^{u_{2}\left(\xi_{2}\right)}\right) \\
-h_{2}\left(\xi_{2}\right) e^{-u_{2}\left(\xi_{2}\right)}=0, \\
-d_{1}\left(\xi_{3}\right)+\beta_{3}\left(\xi_{3}\right) p_{3}\left(e^{u_{2}\left(\xi_{3}-\tau\right)}\right)-a_{31}\left(\xi_{3}\right) e^{u_{3}\left(\xi_{3}\right)}-h_{3}\left(\xi_{3}\right) e^{-u_{3}\left(\xi_{3}\right)}=0
\end{array}\right.
$$

and

$$
\left\{\begin{array}{l}
g_{1}\left(\eta_{1}, e^{u_{1}\left(\eta_{1}\right)}\right)-c_{1}\left(\eta_{1}\right) e^{u_{2}\left(\eta_{1}\right)-u_{1}\left(\eta_{1}\right)} p_{2}\left(e^{u_{1}\left(\eta_{1}\right)}\right)-h_{1}\left(\eta_{1}\right) e^{-u_{1}\left(\eta_{1}\right)}=0, \\
-g_{2}\left(\eta_{2}, e^{u_{2}\left(\eta_{2}\right)}\right)+\beta_{1}\left(\eta_{2}\right) p_{2}\left(e^{u_{1}\left(\eta_{2}-\tau\right)}\right)-\beta_{2}\left(\eta_{2}\right) e^{u_{3}\left(\eta_{2}\right)-u_{2}\left(\eta_{2}\right)} p_{3}\left(e^{u_{2}\left(\eta_{2}\right)}\right) \\
-h_{2}\left(\eta_{2}\right) e^{-u_{2}\left(\eta_{2}\right)}=0, \\
-d_{1}\left(\eta_{3}\right)+\beta_{3}\left(\eta_{3}\right) p_{3}\left(e^{u_{2}\left(\eta_{3}-\tau\right)}\right)-a_{31}\left(\eta_{3}\right) e^{u_{3}\left(\eta_{3}\right)} \\
-h_{3}\left(\eta_{3}\right) e^{-u_{3}\left(\eta_{3}\right)}=0 .
\end{array}\right.
$$

(2.4) implies that

$$
g_{1}\left(\xi_{1}, e^{u_{1}\left(\xi_{1}\right)}\right)>0
$$

whereby along with condition (i) in Theorem 2.2 , we have

$$
u_{1}\left(\xi_{1}\right)<A \text {. }
$$

(2.7) implies that

$$
g_{1}\left(\eta_{1}, 0\right)>g_{1}\left(\eta_{1}, e^{u_{1}\left(\eta_{1}\right)}\right)>h_{1}^{l} e^{-u_{1}\left(\eta_{1}\right)},
$$

that is,

$$
u_{1}\left(\eta_{1}\right)>\ln \left[\frac{h_{1}^{l}}{g_{1}\left(\eta_{1}, 0\right)}\right] \geq \ln \frac{h_{1}^{l}}{a_{1}^{M}}
$$


(2.5) implies that

$$
g_{2}\left(\xi_{2}, e^{u_{2}\left(\xi_{2}\right)}\right)<\beta_{1}^{M} p_{2}\left(e^{A}\right),
$$

whereby along with condition (ii) in Theorem 2.2, we have

$$
u_{2}\left(\xi_{2}\right)<B \text {. }
$$

(2.8) implies that

$$
h_{2}^{l} e^{-u_{2}\left(\eta_{2}\right)} \leq h_{2}\left(\eta_{2}\right) e^{-u_{2}\left(\eta_{2}\right)}<\beta_{1}^{M} p_{2}\left(e^{A}\right),
$$

that is,

$$
u_{2}\left(\eta_{2}\right)>\ln \left(\frac{h_{2}^{l}}{\beta_{1}^{M} p_{2}\left(e^{A}\right)}\right)
$$

(2.6) implies that

$$
a_{31}^{l} e^{u_{3}\left(\xi_{3}\right)} \leq a_{31}\left(\xi_{3}\right) e^{u_{2}\left(\xi_{3}\right)}<\beta_{3}^{M} p_{3}\left(e^{B}\right),
$$

that is,

$$
u_{3}\left(\xi_{3}\right)<\ln \frac{\beta_{3}^{M} p_{3}\left(e^{B}\right)}{a_{31}^{l}} .
$$

(2.9) implies that

$$
\beta_{3}^{M} p_{3}\left(e^{B}\right) \geq \beta_{3}\left(\eta_{3}\right) p_{3}\left(e^{u_{2}\left(\eta_{3}-\tau\right)}\right)>h_{3}^{l} e^{-u_{3}\left(\eta_{3}\right)},
$$

that is,

$$
u_{3}\left(\eta_{3}\right)>\ln \left[\frac{h_{3}^{l}}{\beta_{3}^{M} p_{3}\left(e^{B}\right)}\right] .
$$

(2.4) implies that

$$
e^{u_{1}\left(\xi_{1}\right)} g_{1}\left(\xi_{1}, e^{u_{1}\left(\xi_{1}\right)}\right)<c_{1}^{M} e^{B} p_{2}\left(e^{u_{1}\left(\xi_{1}\right)}\right)+h_{1}^{M},
$$

whereby along with condition (iii) in Theorem 2.2, we have

$$
u_{1}\left(\xi_{1}\right)>\ln l_{+} \quad \text { or } \quad u_{1}\left(\xi_{1}\right)<\ln l_{-} .
$$

Similarly, from (2.7), we have

$$
u_{1}\left(\eta_{1}\right)>\ln l_{+} \quad \text { or } \quad u_{1}\left(\eta_{1}\right)<\ln l_{-} .
$$

(2.5) implies that

$$
-e^{u_{2}\left(\xi_{2}\right)} g_{2}\left(\xi_{2}, e^{u_{2}\left(\xi_{2}\right)}\right)+\beta_{1}\left(\xi_{2}\right) e^{u_{2}\left(\xi_{2}\right)} p_{2}\left(e^{u_{1}\left(\xi_{2}-\tau\right)}\right)-\beta_{2}\left(\xi_{2}\right) e^{u_{3}\left(\xi_{2}\right)} p_{3}\left(e^{u_{2}\left(\xi_{2}\right)}\right)-h_{2}\left(\xi_{2}\right)=0,
$$

that is,

$$
e^{u_{2}\left(\xi_{2}\right)} g_{2}\left(\xi_{2}, e^{u_{2}\left(\xi_{2}\right)}\right)+h_{2}^{M}+\frac{\beta_{2}^{M} \beta_{3}^{M}}{a_{31}^{l}} p_{3}\left(e^{B}\right) p_{3}\left(e^{u_{2}\left(\xi_{2}\right)}\right)>\beta_{1}^{l} e^{u_{2}\left(\xi_{2}\right)} p_{2}\left(\frac{h_{1}^{l}}{a_{1}^{M}}\right),
$$

whereby along with condition (iv) in Theorem 2.2 , we obtain

$$
u_{2}\left(\xi_{2}\right)>\ln u_{+} \text {or } u_{2}\left(\xi_{2}\right)<\ln u_{-} .
$$


Similarly, from (2.8), we have

$$
u_{2}\left(\eta_{2}\right)>\ln u_{+} \text {or } u_{2}\left(\eta_{2}\right)<\ln u_{-} .
$$

(2.6) implies that

$$
a_{31}^{M} e^{2 u_{3}\left(\xi_{3}\right)}+d_{1}^{M} e^{u_{3}\left(\xi_{3}\right)}+h_{3}^{M}>\beta_{3}^{l} p_{3}\left(\frac{h_{2}^{l}}{\beta_{1}^{M} p_{2}\left(e^{A}\right)}\right) e^{u_{3}\left(\xi_{3}\right)},
$$

that is,

$$
u_{3}\left(\xi_{3}\right)>\ln v_{+} \text {or } u_{3}\left(\xi_{3}\right)<\ln v_{-}
$$

where

$$
v_{ \pm}=\frac{\beta_{3}^{l} p_{3}\left(\frac{h_{2}^{l}}{\beta_{1}^{M} p_{2}\left(e^{A}\right)}\right)-d_{1}^{M} \pm \sqrt{\left[\beta_{3}^{l} p_{3}\left(\frac{h_{2}^{l}}{\beta_{1}^{M} p_{2}\left(e^{A}\right)}\right)-d_{1}^{M}\right]^{2}-4 a_{31}^{M} h_{3}^{M}}}{2 a_{31}^{M}} .
$$

Similarly, from (2.9), we obtain

$$
u_{3}\left(\eta_{3}\right)>\ln v_{+} \text {or } u_{3}\left(\eta_{3}\right)<\ln v_{-} .
$$

From (2.10), (2.11), (2.16) and (2.17), we have for $t \in[0, \omega]$,

$$
\ln \frac{h_{1}^{l}}{a_{1}^{M}}<u_{1}(t)<\ln l_{-} \text {or } \ln l_{+}<u_{1}(t)<A .
$$

From (2.12), (2.13), (2.18) and (2.19), we obtain for $t \in[0, \omega]$,

$$
\ln \left(\frac{h_{2}^{l}}{\beta_{1}^{M} p_{2}\left(e^{A}\right)}\right)<u_{2}(t)<\ln u_{-} \text {or } \ln u_{+}<u_{2}(t)<B
$$

From (2.14), (2.15), (2.20) and (2.21), we have for $t \in[0, \omega]$,

$$
\ln \left(\frac{h_{3}^{l}}{\beta_{3}^{M} p_{3}\left(e^{B}\right)}\right)<u_{3}(t)<\ln v_{-} \text {or } \ln v_{+}<u_{3}(t)<\ln \left(\frac{\beta_{3}^{M} p_{3}\left(e^{B}\right)}{a_{31}^{l}}\right) .
$$


Obviously, $\ln l_{ \pm}, \ln u_{ \pm}, \ln v_{ \pm}, \ln \frac{h_{1}^{l}}{a_{1}^{M}}, \ln \left(\frac{h_{2}^{l}}{\beta_{1}^{M} p_{2}\left(e^{A}\right)}\right), \ln \left(\frac{h_{3}^{l}}{\beta_{3}^{M} p_{3}\left(e^{B}\right)}\right), A, B, \ln \left(\frac{\beta_{3}^{M} p_{3}\left(e^{B}\right)}{a_{31}^{l}}\right)$ are independent of $\lambda$. Now we let

$$
\begin{aligned}
& \Omega_{1}=\left\{u=\left(u_{1}, u_{2}, u_{3}\right)^{T} \in X / \begin{array}{l}
u_{1}(t) \in\left(\ln \frac{h_{1}^{l}}{a_{1}^{M}}, \ln l_{-}\right) \\
u_{2}(t) \in\left(\ln \left(\frac{h_{2}^{l}}{\beta_{1}^{M} p_{2}\left(e^{A}\right)}\right), \ln u_{-}\right) \\
u_{3}(t) \in\left(\ln \left(\frac{h_{3}^{l}}{\beta_{3}^{M} p_{3}\left(e^{B}\right)}\right), \ln v_{-}\right)
\end{array}\right\}, \\
& \Omega_{2}=\left\{u=\left(u_{1}, u_{2}, u_{3}\right)^{T} \in X / \begin{array}{l}
u_{1}(t) \in\left(\ln \frac{h_{1}^{l}}{a_{1}^{M}}, \ln l_{-}\right) \\
u_{2}(t) \in\left(\ln u_{+}, B\right) \\
u_{3}(t) \in\left(\ln \left(\frac{h_{3}^{l}}{\beta_{3}^{M} p_{3}\left(e^{B}\right)}\right), \ln v_{-}\right)
\end{array}\right\}, \\
& \Omega_{3}=\left\{u=\left(u_{1}, u_{2}, u_{3}\right)^{T} \in X / \begin{array}{l}
u_{1}(t) \in\left(\ln l_{+}, A\right) \\
u_{2}(t) \in\left(\ln \left(\frac{h_{2}^{l}}{\beta_{1}^{M} p_{2}\left(e^{A}\right)}\right), \ln u_{-}\right) \\
u_{3}(t) \in\left(\ln \left(\frac{h_{3}^{l}}{\beta_{3}^{M} p_{3}\left(e^{B}\right)}\right), \ln v_{-}\right)
\end{array}\right\}, \\
& \Omega_{4}=\left\{u=\left(u_{1}, u_{2}, u_{3}\right)^{T} \in X / \begin{array}{l}
u_{1}(t) \in\left(\ln l_{+}, A\right) \\
u_{2}(t) \in\left(\ln u_{+}, B\right) \\
u_{3}(t) \in\left(\ln \left(\frac{h_{3}^{l}}{\beta_{3}^{M} p_{3}\left(e^{B}\right)}\right), \ln v_{-}\right)
\end{array}\right\}, \\
& \Omega_{5}=\left\{u=\left(u_{1}, u_{2}, u_{3}\right)^{T} \in X / \begin{array}{l}
u_{1}(t) \in\left(\ln \frac{h_{1}^{l}}{a_{1}^{M}}, \ln l_{-}\right) \\
u_{2}(t) \in\left(\ln \left(\frac{h_{2}^{l}}{\beta_{1}^{M} p_{2}\left(e^{A}\right)}\right), \ln u_{-}\right) \\
u_{3}(t) \in\left(\ln v_{+}, \ln \left(\frac{\beta_{3}^{M} p_{3}\left(e^{B}\right)}{a_{31}^{l}}\right)\right)
\end{array}\right\}, \\
& \Omega_{6}=\left\{u=\left(u_{1}, u_{2}, u_{3}\right)^{T} \in X / \begin{array}{l}
u_{1}(t) \in\left(\ln \frac{h_{1}^{l}}{a_{1}^{M}}, \ln l_{-}\right) \\
u_{2}(t) \in\left(\ln u_{+}, B\right) \\
u_{3}(t) \in\left(\ln v_{+}, \ln \left(\frac{\beta_{3}^{M} p_{3}\left(e^{B}\right)}{a_{31}^{l}}\right)\right)
\end{array}\right\}, \\
& \Omega_{7}=\left\{u=\left(u_{1}, u_{2}, u_{3}\right)^{T} \in X / \begin{array}{l}
u_{1}(t) \in\left(\ln l_{+}, A\right) \\
u_{2}(t) \in\left(\ln \left(\frac{h_{2}^{l}}{\beta_{3}^{M} p_{2}\left(e^{A}\right)}\right), \ln u_{-}\right) \\
u_{3}(t) \in\left(\ln v_{+}, \ln \left(\frac{\beta_{3}^{M} p_{3}\left(e^{B}\right)}{a_{31}^{l}}\right)\right)
\end{array}\right\},
\end{aligned}
$$

and

$$
\Omega_{8}=\left\{u=\left(u_{1}, u_{2}, u_{3}\right)^{T} \in X / \begin{array}{l}
u_{1}(t) \in\left(\ln l_{+}, A\right) \\
u_{2}(t) \in\left(\ln u_{+}, B\right) \\
u_{3}(t) \in\left(\ln v_{+}, \ln \left(\frac{\beta_{3}^{M} p_{3}\left(e^{B}\right)}{a_{31}^{l}}\right)\right)
\end{array}\right\} .
$$

Then $\Omega_{i}(i=1,2,3,4,5,6,7,8)$ are bounded open subsets of $X, \Omega_{i} \cap \Omega_{j}=\phi, i \neq j$, $i, j=1,2,3,4,5,6,7,8$, and $\Omega_{i}(i=1,2,3,4,5,6,7,8)$ satisfies requirement (a) in Theorem 2.2.

Next, we prove that (b) of Theorem 2.1 holds, i.e., we prove that when $u \in \partial \Omega_{i} \cap$ $\operatorname{Ker} L=\partial \Omega_{i} \cap R^{3}, i=1,2,3,4,5,6,7,8, Q N u \neq(0,0,0)^{T}$. There exist three points 
$t_{i} \in[0, \omega], i=1,2,3$, such that when $u \in \partial \Omega_{i} \cap R^{3}, i=1,2,3,4,5,6,7,8$,

$$
Q N u=\left[\begin{array}{l}
g_{1}\left(t_{1}, e^{u_{1}}\right)-c_{1}(t) e^{u_{2}-u_{1}} p_{2}\left(e^{u_{1}}\right)-h_{1}\left(t_{1}\right) e^{-u_{1}} \\
-g_{2}\left(t_{2}, e^{u_{2}}\right)+\beta_{1}\left(t_{2}\right) p_{2}\left(e^{u_{1}}\right)-\beta_{2}\left(t_{2}\right) e^{u_{3}-u_{2}} p_{3}\left(e^{u_{2}}\right)-h_{2}\left(t_{2}\right) e^{-u_{2}} \\
-d_{1}\left(t_{3}\right)+\beta_{3}\left(t_{3}\right) p_{3}\left(e^{u_{2}}\right)-a_{31}\left(t_{3}\right) e^{u_{3}}-h_{3}\left(t_{3}\right) e^{-u_{3}}
\end{array}\right] .
$$

If when $u \in \partial \Omega_{i} \cap \operatorname{Ker} L=\partial \Omega_{i} \cap R^{3}, Q N u=(0,0,0)^{T}$, then vector $u$ in $R^{3}$ with $u \in \partial \Omega_{i}, i=1,2,3,4,5,6,7,8$, satisfies

$$
\left\{\begin{array}{l}
g_{1}\left(t_{1}, e^{u_{1}}\right)=c_{1}(t) e^{u_{2}-u_{1}} p_{2}\left(e^{u_{1}}\right)+h_{1}\left(t_{1}\right) e^{-u_{1}} \\
g_{2}\left(t_{2}, e^{u_{2}}\right)+h_{2}\left(t_{2}\right) e^{-u_{2}}+\beta_{2}\left(t_{2}\right) e^{u_{3}-u_{2}} p_{3}\left(e^{u_{2}}\right)=\beta_{1}\left(t_{2}\right) p_{2}\left(e^{u_{1}}\right) \\
d_{1}\left(t_{3}\right)+h_{3}\left(t_{3}\right) e^{-u_{3}}+a_{31}\left(t_{3}\right) e^{u_{3}}=\beta_{3}\left(t_{3}\right) p_{3}\left(e^{u_{2}}\right)
\end{array}\right.
$$

From (2.25)-(2.27), following the arguments of (2.22)-(2.24), we obtain

$$
\begin{gathered}
\ln \frac{h_{1}^{l}}{a_{1}^{M}}<u_{1}<\ln l_{-} \text {or } \ln l_{+}<u_{1}<A, \\
\ln \left(\frac{h_{2}^{l}}{\beta_{1}^{M} p_{2}\left(e^{A}\right)}\right)<u_{2}<\ln u_{-} \text {or } \ln u_{+}<u_{2}<B, \\
\ln \left(\frac{h_{3}^{l}}{\beta_{3}^{M} p_{3}\left(e^{B}\right)}\right)<u_{3}<\ln v_{-} \text {or } \ln v_{+}<u_{3}<\ln \left(\frac{\beta_{3}^{M} p_{3}\left(e^{B}\right)}{a_{31}^{l}}\right) .
\end{gathered}
$$

Thus $u \in \Omega_{1} \cap R^{3}$ or $u \in \Omega_{2} \cap R^{3}$ or $u \in \Omega_{3} \cap R^{3}$ or $u \in \Omega_{4} \cap R^{3}$ or $u \in \Omega_{5} \cap R^{3}$ or $u \in \Omega_{6} \cap R^{3}$, or $u \in \Omega_{7} \cap R^{3}$ or $u \in \Omega_{8} \cap R^{3}$. This leads to a contradiction and proves that (b) in Theorem 2.1 holds.

Finally, we show that (c) in Theorem 2.1 holds; we only prove that when $u \in \partial \Omega_{i} \cap$ $\operatorname{Ker} L=\partial \Omega_{i} \cap R^{3}, i=1,2,3,4,5,6,7,8, \operatorname{deg}\left\{J Q N u, \Omega_{i} \cap \operatorname{Ker} L,(0,0,0)^{T}\right\} \neq(0,0,0)^{T}$. Our proof is broken into two steps.

Step 1. We show that for $i=1,2,3,4,5,6,7,8$,

$$
\begin{aligned}
& \operatorname{deg}\left\{J Q N u, \Omega_{i} \cap \operatorname{Ker} L,(0,0,0)^{T}\right\} \\
= & \operatorname{deg}\left\{\left[g_{1}\left(t_{1}, e^{u_{1}}\right)-c_{1}\left(t_{1}\right) e^{u_{2}-u_{1}} p_{2}\left(e^{u_{1}}\right)-h_{1}\left(t_{1}\right) e^{-u_{1}},-g_{2}\left(t_{2}, e^{u_{2}}\right)\right.\right. \\
& +\beta_{1}\left(t_{2}\right) p_{2}\left(e^{u_{1}}\right)-\beta_{2}\left(t_{2}\right) e^{u_{3}-u_{2}} p_{3}\left(e^{u_{2}}\right)-h_{2}\left(t_{2}\right) e^{-u_{2}},-d_{1}\left(t_{3}\right)+\beta_{3}\left(t_{3}\right) p_{3}\left(e^{u_{2}}\right) \\
& \left.\left.-a_{31}\left(t_{3}\right) e^{u_{3}}-h_{3}\left(t_{3}\right) e^{-u_{3}}\right]^{T}, \Omega_{i} \cap \operatorname{Ker} L,(0,0,0)^{T}\right\} \\
= & \operatorname{deg}\left\{\left[g_{1}\left(t_{1}, e^{u_{1}}\right)-h_{1}\left(t_{1}\right) e^{-u_{1}},-g_{2}\left(t_{2}, e^{u_{2}}\right)\right.\right. \\
& +\beta_{1}\left(t_{2}\right) p_{2}\left(e^{u_{1}}\right)-h_{2}\left(t_{2}\right) e^{-u_{2}},-d_{1}\left(t_{3}\right)+\beta_{3}\left(t_{3}\right) p_{3}\left(e^{u_{2}}\right) \\
& \left.\left.-a_{31}\left(t_{3}\right) e^{u_{3}}-h_{3}\left(t_{3}\right) e^{-u_{3}}\right]^{T}, \Omega_{i} \cap \operatorname{Ker} L,(0,0,0)^{T}\right\} .
\end{aligned}
$$

To this end, we define mapping $\phi_{1}: \operatorname{Dom} L \times[0,1] \rightarrow X$ by

$$
\phi_{1}\left(u_{1}, u_{2}, u_{3}, \mu_{1}\right)=\left[\begin{array}{l}
g_{1}\left(t_{1}, e^{u_{1}}\right)-\mu_{1} c_{1}\left(t_{1}\right) e^{u_{2}-u_{1}} p_{2}\left(e^{u_{1}}\right)-h_{1}\left(t_{1}\right) e^{-u_{1}} \\
-g_{2}\left(t_{2}, e^{u_{2}}\right)+\beta_{1}\left(t_{2}\right) p_{2}\left(e^{u_{1}}\right)-\mu_{1} \beta_{2}\left(t_{2}\right) e^{u_{3}-u_{2}} p_{3}\left(e^{u_{2}}\right)-h_{2}\left(t_{2}\right) e^{-u_{2}} \\
-d_{1}\left(t_{3}\right)+\beta_{3}\left(t_{3}\right) p_{3}\left(e^{u_{2}}\right)-a_{31}\left(t_{3}\right) e^{u_{3}}-h_{3}\left(t_{3}\right) e^{-u_{3}}
\end{array}\right],
$$


where $\mu_{1} \in[0,1]$ is a parameter. When $u \in \partial \Omega_{i} \cap \operatorname{Ker} L=\partial \Omega_{i} \cap R^{3}, u$ is a constant vector in $R^{3}$ with $u \in \partial \Omega_{i}, i=1,2,3,4,5,6,7,8$. We show that when $u \in \partial \Omega_{i} \cap \operatorname{Ker} L=$ $\partial \Omega_{i} \cap \operatorname{Ker} L R^{3}, \phi_{1}\left(u_{1}, u_{2}, u_{3}, \mu_{1}\right) \neq(0,0,0)^{T}$. If it is not true, then constant vector $u$ in $R^{3}$ with $u \in \partial \Omega_{i}(i=1,2,3,4,5,6,7,8)$ satisfies

$$
\left\{\begin{array}{l}
g_{1}\left(t_{1}, e^{u_{1}}\right)-\mu_{1} c_{1}\left(t_{1}\right) e^{u_{2}-u_{1}} p_{2}\left(e^{u_{1}}\right)-h_{1}\left(t_{1}\right) e^{-u_{1}}=0 \\
-g_{2}\left(t_{2}, e^{u_{2}}\right)+\beta_{1}\left(t_{2}\right) p_{2}\left(e^{u_{1}}\right)-\mu_{1} \beta_{2}\left(t_{2}\right) e^{u_{3}-u_{2}} p_{3}\left(e^{u_{2}}\right)-h_{2}\left(t_{2}\right) e^{-u_{2}}=0 \\
-d_{1}\left(t_{3}\right)+\beta_{3}\left(t_{3}\right) p_{3}\left(e^{u_{2}}\right)-a_{31}\left(t_{3}\right) e^{u_{3}}-h_{3}\left(t_{3}\right) e^{-u_{3}}
\end{array}\right.
$$

From (2.28)-(2.30), following the arguments of (2.22)-(2.24) and by magnifying $\mu_{1}$ into 1 or reducing $\mu_{1}$ into 0 , we obtain

$$
\begin{gathered}
\ln \frac{h_{1}^{l}}{a_{1}^{M}}<u_{1}<\ln l_{-} \text {or } \ln l_{+}<u_{1}<A, \\
\ln \left[\frac{h_{2}^{l}}{\beta_{1}^{M} p_{2}\left(e^{A}\right)}\right]<u_{2}<\ln u_{-} \text {or } \ln u_{+}<u_{2}<B, \\
\ln \left[\frac{h_{3}^{l}}{\beta_{3}^{M} p_{3}\left(e^{B}\right)}\right]<u_{3}<\ln v_{-} \text {or } \ln v_{+}<u_{3}<\ln \left[\frac{\beta_{3}^{M} p_{3}\left(e^{B}\right)}{a_{31}^{l}}\right] .
\end{gathered}
$$

Thus $u \in \Omega_{1} \cap R^{3}$ or $u \in \Omega_{2} \cap R^{3}$ or $u \in \Omega_{3} \cap R^{3}$ or $u \in \Omega_{4} \cap R^{3}$ or $u \in \Omega_{5} \cap R^{3}$ or $u \in \Omega_{6} \cap R^{3}$ or $u \in \Omega_{7} \cap R^{3}$ or $u \in \Omega_{8} \cap R^{3}$. This is a contradiction. Therefore, when $u \in$ $\partial \Omega_{i} \cap \operatorname{Ker} L, i=1,2,3,4,5,6,7,8, \phi_{1}\left(u_{1}, u_{2}, u_{3}, \mu_{1}\right) \neq(0,0,0)^{T}$. Based on topological degree theory, we obtain by taking $J$ as the identity mapping since $\operatorname{Ker} L=\operatorname{Im} Q$, for $i=1,2,3,4,5,6,7,8$,

$$
\begin{aligned}
& \operatorname{deg}\left\{J Q N u, \Omega_{i} \cap \operatorname{Ker} L,(0,0,0)^{T}\right\} \\
= & \operatorname{deg}\left\{\phi_{1}\left(u_{1}, u_{2}, u_{3}, 1\right), \Omega_{i} \cap \operatorname{Ker} L,(0,0,0)^{T}\right\} \\
= & \operatorname{deg}\left\{\phi_{1}\left(u_{1}, u_{2},, u_{3}, 0\right), \Omega_{i} \cap \operatorname{Ker} L,(0,0,0)^{T}\right\} \\
= & \operatorname{deg}\left\{\left[g_{1}\left(t_{1}, e^{u_{1}}\right)-h_{1}\left(t_{1}\right) e^{-u_{1}},-g_{2}\left(t_{2}, e^{u_{2}}\right)+\beta_{1}\left(t_{2}\right) p_{2}\left(e^{u_{1}}\right)-h_{2}\left(t_{2}\right) e^{-u_{2}},\right.\right. \\
& \left.\left.-d_{1}\left(t_{3}\right)+\beta_{3}\left(t_{3}\right) p_{3}\left(e^{u_{2}}\right)-a_{31}\left(t_{3}\right) e^{u_{3}}-h_{3}\left(t_{3}\right) e^{-u_{3}}\right]^{T}, \Omega_{i} \cap \operatorname{KerL},(0,0,0)^{T}\right\} .
\end{aligned}
$$

Step 2. We prove that for $i=1,2,3,4,5,6,7,8$,

$$
\begin{aligned}
& \operatorname{deg}\left\{\left[g_{1}\left(t_{1}, e^{u_{1}}\right)-h_{1}\left(t_{1}\right) e^{-u_{1}},-g_{2}\left(t_{2}, e^{u_{2}}\right)+\beta_{1}\left(t_{2}\right) p_{2}\left(e^{u_{1}}\right)-h_{2}\left(t_{2}\right) e^{-u_{2}},\right.\right. \\
& \left.\left.-d_{1}\left(t_{3}\right)+\beta_{3}\left(t_{3}\right) p_{3}\left(e^{u_{2}}\right)-a_{31}\left(t_{3}\right) e^{u_{3}}-h_{3}\left(t_{3}\right) e^{-u_{3}}\right]^{T}, \Omega_{i} \cap \operatorname{Ker} L,(0,0,0)^{T}\right\} \\
= & \operatorname{deg}\left\{\left[a_{1}-b_{1} e^{u_{1}}-h_{1} e^{-u_{1}},-a_{2}-b_{2} e^{u_{2}}+\beta_{1} e^{u_{1}}-h_{2} e^{-u_{2}},\right.\right. \\
& \left.\left.-d_{1}+\beta_{3} e^{u_{2}}-a_{31} e^{u_{3}}-h_{3} e^{-u_{3}}\right]^{T}, \Omega_{i} \cap \operatorname{KerL},(0,0,0)^{T}\right\},
\end{aligned}
$$


where $a_{1}, b_{1}, h_{1}, a_{2}, b_{2}, \beta_{1}, h_{2}, d_{1}, \beta_{3}, a_{31}, h_{3}$ are all some choosen positive constants such that

$$
\begin{aligned}
& \ln \frac{a_{1}}{b_{1}}<A, \quad b_{2} e^{B}>\beta_{1} e^{A}, \quad a_{1} h_{1}^{l}<h_{1} a_{1}^{M}, \beta_{1} h_{2}^{l} e^{A}<\beta_{1}^{M} h_{2} p_{2}\left(e^{A}\right), \\
& a_{31}^{l} \beta_{3} e^{B}<a_{31} \beta_{3}^{M} p_{3}\left(e^{B}\right), \quad h_{3}^{l} \beta_{3} e^{B}<h_{3} \beta_{3}^{M} p_{3}\left(e^{B}\right), \\
& \frac{a_{1}-\sqrt{a_{1}^{2}-4 b_{1} h_{1}}}{2 b_{1}}<l_{-}<l_{+}<\frac{a_{1}+\sqrt{a_{1}^{2}-4 b_{1} h_{1}}}{2 b_{1}}, \\
& \frac{\frac{\beta_{1} h_{1}^{l}}{a_{1}^{M}}-a_{2}-\sqrt{\left(\frac{\beta_{1} h_{1}^{l}}{a_{1}^{M}}-a_{2}\right)^{2}-4 b_{2} h_{2}}}{2 b_{2}}<u_{-}, \\
& u_{+}<\frac{\frac{\beta_{1} h_{1}^{l}}{a_{1}^{M}}-a_{2}+\sqrt{\left(\frac{\beta_{1} h_{1}^{l}}{a_{1}^{M}}-a_{2}\right)^{2}-4 b_{2} h_{2}}}{2 b_{2}}, \\
& \frac{\frac{\beta_{3} h_{2}^{l}}{\beta_{1}^{M} p_{2}\left(e^{A}\right)}-d_{1}-\sqrt{\left(\frac{\beta_{3} h_{2}^{l}}{\beta_{1}^{M} p_{2}\left(e^{A}\right)}-d_{1}\right)^{2}-4 a_{31} h_{3}}}{2 a_{31}}<v_{-}, \\
& v_{+}<\frac{\frac{\beta_{3} h_{2}^{l}}{\beta_{1}^{M} p_{2}\left(e^{A}\right)}-d_{1}+\sqrt{\left(\frac{\beta_{3} h_{2}^{l}}{\beta_{1}^{M} p_{2}\left(e^{A}\right)}-d_{1}\right)^{2}-4 a_{31} h_{3}}}{2 a_{31}} .
\end{aligned}
$$

To this end, we define mapping $\phi_{2}: D o m L \times[0,1] \rightarrow X$ by

$$
\begin{aligned}
\phi_{2}\left(u_{1}, u_{2}, u_{3}, \mu_{2}\right)= & \mu_{2}\left[\begin{array}{l}
a_{1}-b_{1} e^{u_{1}}-h_{1} e^{-u_{1}} \\
-a_{2}-b_{2} e^{u_{2}}+\beta_{1} e^{u_{1}}-h_{2} e^{-u_{2}} \\
-d_{1}+\beta_{3} e^{u_{2}}-a_{31} e^{u_{3}}-h_{3} e^{-u_{3}}
\end{array}\right] \\
& +\left(1-\mu_{2}\right)\left(\begin{array}{l}
g_{1}\left(t_{1}, e^{u_{1}}\right)-h_{1}\left(t_{1}\right) e^{-u_{1}} \\
-g_{2}\left(t_{2}, e^{u_{2}}\right)+\beta_{1}\left(t_{2}\right) p_{2}\left(e^{u_{1}}\right)-h_{2}\left(t_{2}\right) e^{-u_{2}} \\
-d_{1}\left(t_{3}\right)+\beta_{3}\left(t_{3}\right) p_{3}\left(e^{u_{2}}\right)-a_{31}\left(t_{3}\right) e^{u_{3}}-h_{3}\left(t_{3}\right) e^{-u_{3}}
\end{array}\right),
\end{aligned}
$$

where $\mu_{2} \in[0,1]$ is a parameter. We show that when $u \in \partial \Omega_{i} \cap \operatorname{Ker} L=\partial \Omega_{i} \cap R^{3}$, $\phi_{2}\left(u_{1}, u_{2}, u_{3}, \mu_{2}\right) \neq(0,0,0)^{T}$. If the conclusion is not true, i.e., constant vector $u$ in $R^{3}$ with $u \in \partial \Omega_{i}, i=1,2,3,4,5,6,7,8$, satisfies $\phi_{2}\left(u_{1}, u_{2}, u_{3}, \mu_{2}\right)=(0,0,0)^{T}$, then

$$
\left\{\begin{array}{l}
\mu_{2}\left(a_{1}-b_{1} e^{u_{1}}-h_{1} e^{-u_{1}}\right)+\left(1-\mu_{2}\right)\left(g_{1}\left(t_{1}, e^{u_{1}}\right)-h_{1}\left(t_{1}\right) e^{-u_{1}}\right)=0, \\
\mu_{2}\left(-a_{2}-b_{2} e^{u_{2}}+\beta_{1} e^{u_{1}}-h_{2} e^{-u_{2}}\right)+\left(1-\mu_{2}\right)\left(-g_{2}\left(t_{2}, e^{u_{2}}\right)+\beta_{1}\left(t_{2}\right) p_{2}\left(e^{u_{1}}\right)\right. \\
\left.\quad-h_{2}\left(t_{2}\right) e^{-u_{2}}\right)=0, \\
\mu_{2}\left(-d_{1}+\beta_{3} e^{u_{2}}-a_{31} e^{u_{3}}-h_{3} e^{-u_{3}}\right)+\left(1-\mu_{2}\right)\left(-d_{1}\left(t_{3}\right)+\beta_{3}\left(t_{3}\right) p_{3}\left(e^{u_{2}}\right)\right) \\
-a_{31}\left(t_{3}\right) e^{u_{3}}-h_{3}\left(t_{3}\right) e^{-u_{3}}=0 .
\end{array}\right.
$$


We make the following claims.

Claim 1. $u_{1}<A$.

Otherwise, $u_{1} \geq A$. From condition (i) in Theorem 2.2, we have

$$
\begin{aligned}
& \left(1-\mu_{2}\right)\left(g_{1}\left(t_{1}, e^{u_{1}}\right)-h_{1}\left(t_{1}\right) e^{-u_{1}}\right)+\mu_{2}\left(a_{1}-b_{1} e^{u_{1}}-h_{1} e^{-u_{1}}\right) \\
\leq & \left(1-\mu_{2}\right)\left(-h_{1}\left(t_{1}\right) e^{-u_{1}}\right)+\mu_{2}\left(a_{1}-b_{1} e^{A}\right) \\
< & 0 .
\end{aligned}
$$

This contradicts (2.31). Hence Claim 1 holds.

Claim 2. $u_{1}>\ln \frac{h_{1}^{l}}{a_{1}^{M}}$.

Otherwise, $u_{1} \leq \ln \frac{h_{1}^{l}}{a_{1}^{M}}$. Then

$$
\begin{aligned}
& \left(1-\mu_{2}\right)\left(g_{1}\left(t_{1}, e^{u_{1}}\right)-h_{1}\left(t_{1}\right) e^{-u_{1}}\right)++\mu_{2}\left(a_{1}-b_{1} e^{u_{1}}-h_{1} e^{-u_{1}}\right) \\
< & \left(1-\mu_{2}\right)\left(g_{1}\left(t_{1}, 0\right)-h_{1}^{l} e^{-u_{1}}\right)+\mu_{2}\left(a_{1}-h_{1} e^{-u_{1}}\right) \\
< & \left(1-\mu_{2}\right)\left(a_{1}^{M}-h_{1}^{l} e^{-u_{1}}\right)+\mu_{2}\left(a_{1}-h_{1} e^{-u_{1}}\right) \\
< & 0 .
\end{aligned}
$$

This contradicts (2.31). Hence Claim 2 holds.

Claim 3. $u_{2}<B$.

Otherwise, $u_{2} \geq B$. From condition (ii) in Theorem 2.2, we obtain

$$
\begin{aligned}
& \left(1-\mu_{2}\right)\left(-g_{2}\left(t_{2}, e^{u_{2}}\right)+\beta_{1}\left(t_{2}\right) p_{2}\left(e^{u_{1}}\right)-h_{2}\left(t_{2}\right) e^{-u_{2}}\right) \\
& +\mu_{2}\left(-a_{2}-b_{2} e^{u_{2}}+\beta_{1} e^{u_{1}}-h_{2} e^{-u_{2}}\right) \\
< & \left(1-\mu_{2}\right)\left(-g_{2}\left(t_{2}, e^{u_{2}}\right)+\beta_{1}^{M} p_{2}\left(e^{A}\right)\right)+\mu\left(-b_{2} e^{u_{2}}+\beta_{1} e^{u_{1}}\right) \\
< & \mu\left(-b_{2} e^{B}+\beta_{1} e^{A}\right) \\
< & 0 .
\end{aligned}
$$

This contradicts (2.32). Hence Claim 3 holds.

Claim 4. $u_{2}>\ln \left[\frac{h_{2}^{l}}{\beta_{1}^{M} p_{2}\left(e^{A}\right)}\right]$.

Otherwise, $u_{2} \leq \ln \left[\frac{h_{2}^{l}}{\beta_{1}^{M} p_{2}\left(e^{A}\right)}\right]$. Then

$$
\begin{aligned}
& \left(1-\mu_{2}\right)\left(-g_{2}\left(t_{2}, e^{u_{2}}\right)+\beta_{1}\left(t_{2}\right) p_{2}\left(e^{u_{1}}\right)-h_{2}\left(t_{2}\right) e^{-u_{2}}\right) \\
& +\mu_{2}\left(-a_{2}-b_{2} e^{u_{2}}+\beta_{1} e^{u_{1}}-h_{2} e^{-u_{2}}\right) \\
\leq & \left(1-\mu_{2}\right)\left(\beta_{1}^{M} p_{2}\left(e^{A}\right)\right)+\mu_{2}\left(\beta_{1} e^{u_{1}}-h_{2} e^{-u_{2}}\right) \\
< & \left(1-\mu_{2}\right)\left(\beta_{1}^{M} p_{2}\left(e^{A}\right)\right)+\mu_{2}\left(\beta_{1} e^{A}-h_{2} \frac{\beta_{1}^{M} p_{2}\left(e^{A}\right)}{h_{2}^{l}}\right) \\
< & 0 .
\end{aligned}
$$

This contradicts (2.32). Hence Claim 4 holds.

Claim 5. $u_{3}<\ln \left[\frac{\beta_{3}^{M} p_{3}\left(e^{B}\right)}{a_{31}^{l}}\right]$. 
Otherwise, $u_{3} \geq \ln \left[\frac{\beta_{3}^{M} p_{3}\left(e^{B}\right)}{a_{31}^{l}}\right]$. We have

$$
\begin{aligned}
& \left(1-\mu_{2}\right)\left(-d_{1}\left(t_{3}\right)+\beta_{3}\left(t_{3}\right) p_{3}\left(e^{u_{2}}\right)-a_{31}\left(t_{3}\right) e^{u_{3}}-h_{3}\left(t_{3}\right) e^{-u_{3}}\right) \\
& +\mu_{2}\left(-d_{1}+\beta_{3} e^{u_{2}}-a_{31} e^{u_{3}}-h_{3} e^{-u_{3}}\right) \\
\leq & \left(1-\mu_{2}\right)\left(\beta_{3}^{M} p_{3}\left(e^{B}\right)-a_{31}^{l} e^{u_{3}}\right)+\mu_{2}\left(\beta_{3} e^{B}-a_{31} \frac{\beta_{3}^{M} p_{3}\left(e^{B}\right)}{a_{31}^{l}}\right) \\
< & 0 .
\end{aligned}
$$

This contradicts (2.33). Hence Claim 5 holds.

Claim 6. $u_{3}>\ln \left[\frac{h_{3}^{l}}{\beta_{3}^{M} p_{3}\left(e^{B}\right)}\right]$.

Otherwise, $u_{3} \leq \ln \left[\frac{h_{3}^{l}}{\beta_{3}^{M} p_{3}\left(e^{B}\right)}\right]$. Since

$$
\begin{aligned}
& \left(1-\mu_{2}\right)\left(-d_{1}\left(t_{3}\right)+\beta_{3}\left(t_{3}\right) p_{3}\left(e^{u_{2}}\right)-a_{31}\left(t_{3}\right) e^{u_{3}}-h_{3}\left(t_{3}\right) e^{-u_{3}}\right) \\
& +\mu_{2}\left(-d_{1}+\beta_{3} e^{u_{2}}-a_{31} e^{u_{3}}-h_{3} e^{-u_{3}}\right) \\
\leq & \left.\left(1-\mu_{2}\right)\left(\beta_{3}^{M} p_{3}\left(e^{B}\right)-h_{3}^{l} e^{-u_{3}}\right)\right)+\mu_{2}\left(\beta_{3} e^{B}-h_{3} e^{-u_{3}}\right) \\
< & \mu_{2}\left(\beta_{3} e^{B}-h_{3} \frac{\beta_{3}^{M} p_{3}\left(e^{B}\right)}{h_{3}^{l}}\right) \\
< & 0 .
\end{aligned}
$$

This contradicts (2.33). Hence Claim 6 holds.

Claim 7. $u_{1}>\ln l_{+}$or $u_{1}<\ln l_{-}$.

If it is not true, then $\ln l_{-} \leq u_{1} \leq \ln l_{+}$. Thus from condition (iii) in Theorem 2.2, we have

$$
\begin{aligned}
& \left(1-\mu_{2}\right)\left(g_{1}\left(t_{1}, e^{u_{1}}\right)-h_{1}\left(t_{1}\right) e^{-u_{1}}\right)+\mu_{2}\left(a_{1}-b_{1} e^{u_{1}}-h_{1} e^{-u_{1}}\right) \\
= & \left(1-\mu_{2}\right) e^{-u_{1}}\left(e^{u_{1}} g_{1}\left(t_{1}, e^{u_{1}}\right)-h_{1}\left(t_{1}\right)\right)+\mu_{2} e^{-u_{1}}\left(a_{1} e^{u_{1}}-b_{1} e^{2 u_{1}}-h_{1}\right) \\
\geq & \left(1-\mu_{2}\right) e^{-u_{1}}\left(e^{u_{1}} g_{1}\left(t_{1}, e^{u_{1}}\right)-h_{1}^{M}\right)+\mu_{2} e^{-u_{1}}\left(a_{1} e^{u_{1}}-b_{1} e^{2 u_{1}}-h_{1}\right) \\
> & \mu_{2} e^{-u_{1}}\left(a_{1} e^{u_{1}}-b_{1} e^{2 u_{1}}-h_{1}\right) \\
> & 0 .
\end{aligned}
$$

This contradicts (2.31). Hence Claim 7 holds.

Claim 8. $u_{2}>\ln u_{+}$or $u_{2}<\ln u_{-}$.

If the conclusion is not true, then $\ln u_{-} \leq u_{2} \leq \ln u_{+}$. Thus from condition (iv) in Theorem 2.2, we obtain

$$
\begin{aligned}
& \left(1-\mu_{2}\right)\left(-g_{2}\left(t_{2}, e^{u_{2}}\right)+\beta_{1}\left(t_{2}\right) p_{2}\left(e^{u_{1}}\right)-h_{2}\left(t_{2}\right) e^{-u_{2}}\right) \\
& +\mu_{2}\left(-a_{2}-b_{2} e^{u_{2}}+\beta_{1} e^{u_{1}}-h_{2} e^{-u_{2}}\right) \\
= & \left(1-\mu_{2}\right) e^{-u_{2}}\left(-e^{u_{2}} g_{2}\left(t_{2}, e^{u_{2}}\right)+e^{u_{2}} \beta_{1}\left(t_{2}\right) p_{2}\left(e^{u_{1}}\right)-h_{2}\left(t_{2}\right)\right) \\
& +\mu_{2} e^{-u_{2}}\left(-a_{2} e^{u_{2}}-b_{2} e^{2 u_{2}}+\beta_{1} e^{u_{1}+u_{2}}-h_{2}\right) \\
> & \left(1-\mu_{2}\right) e^{-u_{2}}\left(-e^{u_{2}} g_{2}\left(t_{2}, e^{u_{2}}\right)+\beta_{1}^{l} e^{u_{2}} p_{2}\left(\frac{h_{1}^{l}}{a_{1}^{M}}\right)-h_{2}^{M}\right) \\
& +\mu_{2} e^{-u_{2}}\left(-a_{2} e^{u_{2}}-b_{2} e^{2 u_{2}}+\frac{\beta_{1} h_{1}^{l} e^{u_{2}}}{a_{1}^{M}}-h_{2}\right) \\
> & 0 .
\end{aligned}
$$


This contradicts (2.32). Hence Claim 8 holds.

Claim 9. $u_{3}>\ln v_{+}$or $u_{3}<\ln v_{-}$.

If the conclusion is not true, then $\ln v_{-} \leq u_{3} \leq \ln v_{+}$. Thus from condition (v) in Theorem 2.2, we have

$$
\begin{aligned}
& \mu_{2}\left(-d_{1}+\beta_{3} e^{u_{2}}-a_{31} e^{u_{3}}-h_{3} e^{-u_{3}}\right)+\left(1-\mu_{2}\right)\left(-d_{1}\left(t_{3}\right)\right. \\
& \left.+\beta_{3}\left(t_{3}\right) p_{3}\left(e^{u_{2}}\right)-a_{31}\left(t_{3}\right) e^{u_{3}}-h_{3}\left(t_{3}\right) e^{-u_{3}}\right) \\
> & \mu_{2} e^{-u_{3}}\left(-d_{1} e^{u_{3}}+\beta_{3} e^{u_{2}+u_{3}}-a_{31} e^{2 u_{3}}-h_{3}\right) \\
& +\left(1-\mu_{2}\right)\left(-d_{1}^{M} e^{u_{3}}+\beta_{3}^{l} p_{3}\left(\frac{h_{2}^{l}}{\beta_{1}^{M} p_{2}\left(e^{A}\right)}\right) e^{u_{3}}-a_{31}^{M} e^{2 u_{3}}-h_{3}^{M}\right) \\
> & \mu_{2} e^{-u_{3}}\left(-d_{1} e^{u_{3}}+\frac{\beta_{3} h_{2}^{l} e^{u_{3}}}{\beta_{1}^{M} p_{2}\left(e^{A}\right)}-a_{31} e^{2 u_{3}}-h_{3}\right) \\
> & 0 .
\end{aligned}
$$

This contradicts (2.33). Hence Claim 9 holds.

From Claim 1-Claim 9, we have

$$
\begin{gathered}
\ln \frac{h_{1}^{l}}{a_{1}^{M}}<u_{1}<\ln l_{-} \text {or } \ln l_{+}<u_{1}<A, \\
\ln \left[\frac{h_{2}^{l}}{\beta_{1}^{M} p_{2}\left(e^{A}\right)}\right]<u_{2}<\ln u_{-} \text {or } \ln u_{+}<u_{2}<B, \\
\ln \left[\frac{h_{3}^{l}}{\beta_{3}^{M} p_{3}\left(e^{B}\right)}\right]<u_{3}<\ln v_{-} \text {or } \ln v_{+}<u_{3}<\ln \left[\frac{\beta_{3}^{M} p_{3}\left(e^{B}\right)}{a_{31}^{l}}\right] .
\end{gathered}
$$

Thus $u \in \Omega_{1} \cap R^{3}$ or $u \in \Omega_{2} \cap R^{3}$ or $u \in \Omega_{3} \cap R^{3}$ or $u \in \Omega_{4} \cap R^{3} u \in \Omega_{5} \cap R^{3}$ or $u \in \Omega_{6} \cap R^{3}$ or $u \in \Omega_{7} \cap R^{3}$ or $u \in \Omega_{8} \cap R^{3}$, which is a contradiction. Hence when $u \in \partial \Omega_{i} \cap \operatorname{Ker} L=\partial \Omega_{i} \cap R^{3}, i=1,2,3,4,5,6,7,8$, then $\phi_{2}\left(u_{1}, u_{2}, u_{3}, \mu_{2}\right) \neq(0,0,0)^{T}$. Therefore, according to topological degree theory, we obtain for $i=1,2,3,4,5,6,7,8$,

$$
\begin{aligned}
& \operatorname{deg}\left\{\left[g_{1}\left(t_{1}, e^{u_{1}}\right)-h_{1}\left(t_{1}\right) e^{-u_{1}},-g_{2}\left(t_{2}, e^{u_{2}}\right)+\beta_{1}\left(t_{2}\right) p_{2}\left(e^{u_{1}}\right)-h_{2}\left(t_{2}\right) e^{-u_{2}},\right.\right. \\
& \left.\left.\left.\quad-d_{1}\left(t_{3}\right)+\beta_{3}\left(t_{3}\right) p_{3}\left(e^{u_{2}}\right)-a_{31}\left(t_{3}\right) e^{u_{3}}-h_{3}\left(t_{3}\right)\right) e^{-u_{3}}\right]^{T}, \Omega_{i} \cap \operatorname{Ker} L,(0,0,0)^{T}\right\} \\
= & \operatorname{deg}\left\{\phi_{2}\left(u_{1}, u_{2}, u_{3}, 0\right), \Omega_{i} \cap \operatorname{Ker} L,(0,0,0)^{T}\right\} \\
= & \operatorname{deg}\left\{\phi_{2}\left(u_{1}, u_{2}, u_{3}, 1\right), \Omega_{i} \cap \operatorname{Ker} L,(0,0,0)^{T}\right\} \\
= & \operatorname{deg}\left\{\left[a_{1}-b_{1} e^{u_{1}}-h_{1} e^{-u_{1}},-a_{2}-b_{2} e^{u_{2}}+\beta_{1} e^{u_{1}}-h_{2} e^{-u_{2}},\right.\right. \\
& \left.\left.-d_{1}+\beta_{3} e^{u_{2}}-a_{31} e^{u_{3}}-h_{3} e^{-u_{3}}\right]^{T}, \Omega_{i} \cap \operatorname{KerL},(0,0,0)^{T}\right\} .
\end{aligned}
$$

From Step 1 and Step 2, we have for $i=1,2,3,4,5,6,7,8$,

$$
\begin{aligned}
& \operatorname{deg}\left\{J Q N u, \Omega_{i} \cap \operatorname{KerL},(0,0,0)^{T}\right\} \\
= & \operatorname{deg}\left\{\left[a_{1}-b_{1} e^{u_{1}}-h_{1} e^{-u_{1}},-a_{2}-b_{2} e^{u_{2}}+\beta_{1} e^{u_{1}}-h_{2} e^{-u_{2}},\right.\right. \\
& \left.\left.-d_{1}+\beta_{3} e^{u_{2}}-a_{31} e^{u_{3}}-h_{3} e^{-u_{3}}\right]^{T}, \Omega_{i} \cap \operatorname{Ker} L,(0,0,0)^{T}\right\} .
\end{aligned}
$$


Note that the system of algebraic equations:

$$
\left\{\begin{array}{l}
a_{1}-b_{1} e^{x}-h_{1} e^{-x}=0, \\
-a_{2}-b_{2} e^{y}+\beta_{1} e^{x}-h_{2} e^{-y}=0 \\
-d_{1}+\beta_{3} e^{y}-a_{31} e^{z}-h_{3} e^{-z}=0
\end{array}\right.
$$

has eight distinct solutions:

$$
\begin{aligned}
& \left(x_{1}^{*}, y_{1}^{*}, z_{1}^{*}\right)=\left(\ln x_{-}, \ln y_{+}^{-}, \ln \left[\frac{\beta_{3} y_{+}^{-}-d_{1}+\sqrt{\left(\beta_{3} y_{+}^{-}-d_{1}\right)^{2}-4 h_{3} a_{31}}}{2 a_{31}}\right]\right), \\
& \left(x_{2}^{*}, y_{2}^{*}, z_{2}^{*}\right)=\left(\ln x_{-}, \ln y_{+}^{-}, \ln \left[\frac{\beta_{3} y_{+}^{-}-d_{1}-\sqrt{\left(\beta_{3} y_{+}^{-}-d_{1}\right)^{2}-4 h_{3} a_{31}}}{2 a_{31}}\right]\right), \\
& \left(x_{3}^{*}, y_{3}^{*}, z_{3}^{*}\right)=\left(\ln x_{-}, \ln y_{-}^{-}, \ln \left[\frac{\beta_{3} y_{-}^{-}-d_{1}+\sqrt{\left(\beta_{3} y_{-}^{-}-d_{1}\right)^{2}-4 h_{3} a_{31}}}{2 a_{31}}\right]\right), \\
& \left(x_{4}^{*}, y_{4}^{*}, z_{4}^{*}\right)=\left(\ln x_{-}, \ln y_{-}^{-}, \ln \left[\frac{\beta_{3} y_{-}^{-}-d_{1}-\sqrt{\left(\beta_{3} y_{-}^{-}-d_{1}\right)^{2}-4 h_{3} a_{31}}}{2 a_{31}}\right]\right), \\
& \left(x_{5}^{*}, y_{5}^{*}, z_{5}^{*}\right)=\left(\ln x_{+}, \ln y_{+}^{+}, \ln \left[\frac{\beta_{3} y_{+}^{+}-d_{1}+\sqrt{\left(\beta_{3} y_{+}^{+}-d_{1}\right)^{2}-4 h_{3} a_{31}}}{2 a_{31}}\right]\right), \\
& \left(x_{6}^{*}, y_{6}^{*}, z_{6}^{*}\right)=\left(\ln x_{+}, \ln y_{+}^{+}, \ln \left[\frac{\beta_{3} y_{+}^{+}-d_{1}-\sqrt{\left(\beta_{3} y_{+}^{+}-d_{1}\right)^{2}-4 h_{3} a_{31}}}{2 a_{31}}\right]\right), \\
& \left(x_{7}^{*}, y_{7}^{*}, z_{7}^{*}\right)=\left(\ln x_{+}, \ln y_{-}^{+}, \ln \left[\frac{\beta_{3} y_{-}^{+}-d_{1}+\sqrt{\left(\beta_{3} y_{-}^{+}-d_{1}\right)^{2}-4 h_{3} a_{31}}}{2 a_{31}}\right]\right), \\
& \left(x_{8}^{*}, y_{8}^{*}, z_{8}^{*}\right)=\left(\ln x_{+}, \ln y_{-}^{+}, \ln \left[\frac{\beta_{3} y_{-}^{+}-d_{1}-\sqrt{\left(\beta_{3} y_{-}^{+}-d_{1}\right)^{2}-4 h_{3} a_{31}}}{2 a_{31}}\right]\right),
\end{aligned}
$$

where

$$
\begin{gathered}
x_{ \pm}=\frac{a_{1} \pm \sqrt{a_{1}^{2}-4 b_{1} h_{1}}}{2 b_{1}}, \\
y_{ \pm}^{-}=\frac{\beta_{1} x_{-}-a_{2} \pm \sqrt{\left(\beta_{1} x_{-}-a_{2}\right)^{2}-4 b_{2} h_{2}}}{2 b_{2}} \\
y_{ \pm}^{+}=\frac{\beta_{1} x_{+}-a_{2} \pm \sqrt{\left(\beta_{1} x_{+}-a_{2}\right)^{2}-4 b_{2} h_{2}}}{2 b_{2}} .
\end{gathered}
$$


It is not difficult to verify that

$$
\begin{aligned}
& \ln l_{+}<\ln x_{+}<A, \\
& \ln \frac{h_{1}^{l}}{a_{1}^{M}}<\ln x_{-}<\ln l_{-}, \\
& \ln u_{+}<\ln y_{+}^{+}<B, \\
& \ln u_{+}<\ln y_{+}^{-}<B \text {, } \\
& \ln \left[\frac{h_{2}^{l}}{\beta_{1}^{M} p_{2}\left(e^{A}\right)}\right]<\ln y_{-}^{+}<\ln u_{-} \text {, } \\
& \ln \left[\frac{h_{2}^{l}}{\beta_{1}^{M} p_{2}\left(e^{A}\right)}\right]<\ln y_{-}^{-}<\ln u_{-}, \\
& \ln v_{+}<\ln \left[\frac{\beta_{3} y_{+}^{+}-d_{1}+\sqrt{\left(\beta_{3} y_{+}^{+}-d_{1}\right)^{2}-4 h_{3} a_{31}}}{2 a_{31}}\right]<\ln \left(\frac{\beta_{3}^{M} p_{3}\left(e^{B}\right)}{a_{31}^{l}}\right) \text {, } \\
& \ln v_{+}<\ln \left[\frac{\beta_{3} y_{+}^{-}-d_{1}+\sqrt{\left(\beta_{3} y_{+}^{-}-d_{1}\right)^{2}-4 h_{3} a_{31}}}{2 a_{31}}\right]<\ln \left(\frac{\beta_{3}^{M} p_{3}\left(e^{B}\right)}{a_{31}^{l}}\right) \text {, } \\
& \ln \left(\frac{h_{3}^{l}}{\beta_{3}^{M} p_{3}\left(e^{B}\right)}\right)<\ln \left[\frac{\beta_{3} y_{-}^{-}-d_{1}+\sqrt{\left(\beta_{3} y_{-}^{-}-d_{1}\right)^{2}-4 h_{3} a_{31}}}{2 a_{31}}\right]<\ln v_{-}, \\
& \ln \left(\frac{h_{3}^{l}}{\beta_{3}^{M} p_{3}\left(e^{B}\right)}\right)<\ln \left[\frac{\beta_{3} y_{-}^{+}-d_{1}+\sqrt{\left(\beta_{3} y_{-}^{+}-d_{1}\right)^{2}-4 h_{3} a_{31}}}{2 a_{31}}\right]<\ln v_{-}, \\
& \ln \left(\frac{h_{3}^{l}}{\beta_{3}^{M} p_{3}\left(e^{B}\right)}\right)<\ln \left[\frac{\beta_{3} y_{+}^{+}-d_{1}+\sqrt{\left(\beta_{3} y_{+}^{+}-d_{1}\right)^{2}-4 h_{3} a_{31}}}{2 a_{31}}\right]<\ln v_{-}, \\
& \ln \left(\frac{h_{3}^{l}}{\beta_{3}^{M} p_{3}\left(e^{B}\right)}\right)<\ln \left[\frac{\beta_{3} y_{+}^{-}-d_{1}+\sqrt{\left(\beta_{3} y_{+}^{-}-d_{1}\right)^{2}-4 h_{3} a_{31}}}{2 a_{31}}\right]<\ln v_{-}, \\
& \ln v_{+}<\ln \left[\frac{\beta_{3} y_{-}^{-}-d_{1}+\sqrt{\left(\beta_{3} y_{-}^{-}-d_{1}\right)^{2}-4 h_{3} a_{31}}}{2 a_{31}}\right]<\ln \left(\frac{\beta_{3}^{M} p_{3}\left(e^{B}\right)}{a_{31}^{l}}\right) \text {, } \\
& \ln v_{+}<\ln \left[\frac{\beta_{3} y_{-}^{+}-d_{1}+\sqrt{\left(\beta_{3} y_{-}^{+}-d_{1}\right)^{2}-4 h_{3} a_{31}}}{2 a_{31}}\right]<\ln \left(\frac{\beta_{3}^{M} p_{3}\left(e^{B}\right)}{a_{31}^{l}}\right) \text {. }
\end{aligned}
$$

Hence

$\left(x_{1}^{*}, y_{1}^{*}, z_{1}^{*}\right) \in \Omega_{6},\left(x_{2}^{*}, y_{2}^{*}, z_{2}^{*}\right) \in \Omega_{2},\left(x_{3}^{*}, y_{3}^{*}, z_{3}^{*}\right) \in \Omega_{5},\left(x_{4}^{*}, y_{4}^{*}, z_{4}^{*}\right) \in \Omega_{1},\left(x_{5}^{*}, y_{5}^{*}, z_{5}^{*}\right) \in \Omega_{8}$, $\left(x_{6}^{*}, y_{6}^{*}, z_{6}^{*}\right) \in \Omega_{4},\left(x_{7}^{*}, y_{7}^{*}, z_{7}^{*}\right) \in \Omega_{7},\left(x_{8}^{*}, y_{8}^{*}, z_{8}^{*}\right) \in \Omega_{3}$. 
A direct computation gives for $i=1, \ldots, 8$,

$$
\begin{aligned}
& \operatorname{deg}\left\{J Q N u, \Omega_{i} \cap \operatorname{Ker} L,(0,0,0)^{T}\right\} \\
= & \operatorname{sign}\left|\begin{array}{ccc}
-b_{1} x^{*}+\frac{h_{1}}{x^{*}} & 0 & 0 \\
\beta_{1} x^{*} & -b_{2} y^{*}+\frac{h_{2}}{y^{*}} & 0 \\
0 & \beta_{3} y^{*} & -a_{31} z^{*}+\frac{h_{3}}{z^{*}}
\end{array}\right| /\left(x^{*}, y^{*}, z^{*}\right) \\
= & \left(e^{x_{i}^{*}}, e^{y_{i}^{*}}, e^{z_{i}^{*}}\right) \\
= & -\operatorname{sign}\left[\left(2 b_{1} x^{*}-a_{1}\right)\left(b_{2}\left(y^{*}\right)^{2}-h_{2}\right)\left(a_{31}\left(z^{*}\right)^{2}-h_{3}\right)\right] /\left(x^{*}, y^{*}, z^{*}\right) \\
= & \left(e^{x_{i}^{*}}, e^{y_{i}^{*}}, e^{z_{i}^{*}}\right) .
\end{aligned}
$$

Therefore

$$
\begin{aligned}
& \operatorname{deg}\left\{J Q N u, \Omega_{1} \cap \operatorname{KerL},(0,0,0)^{T}\right\} \\
& =\operatorname{sign}\left\{\sqrt{a_{1}^{2}-4 b_{1} h_{1}}\left[\left(\beta_{1} x_{-}-a_{2}-\sqrt{\left(\beta_{1} x_{-}-a_{2}\right)^{2}-4 b_{2} h_{2}}\right)^{2}-4 b_{2} h_{2}\right]\right. \\
& \left.\times\left[\left(\beta_{3} y_{-}^{-}-d_{1}-\sqrt{\left(\left(\beta_{3} y_{-}^{-}-d_{1}\right)^{2}-4 h_{3} a_{31}\right)}\right)^{2}-4 h_{3} a_{31}\right]\right\} \\
& =1 \text {, } \\
& \operatorname{deg}\left\{J Q N u, \Omega_{2} \cap \operatorname{KerL},(0,0,0)^{T}\right\} \\
& =\operatorname{sign}\left\{\sqrt{a_{1}^{2}-4 b_{1} h_{1}}\left[\left(\beta_{1} x_{-}-a_{2}+\sqrt{\left(\beta_{1} x_{-}-a_{2}\right)^{2}-4 b_{2} h_{2}}\right)^{2}-4 b_{2} h_{2}\right]\right. \\
& \left.\times\left[\left(\beta_{3} y_{+}^{-}-d_{1}-\sqrt{\left(\left(\beta_{3} y_{+}^{-}-d_{1}\right)^{2}-4 h_{3} a_{31}\right)}\right)^{2}-4 a_{31} h_{3}\right]\right\} \\
& =-1 \text {, } \\
& \operatorname{deg}\left\{J Q N u, \Omega_{3} \cap \operatorname{Ker} L,(0,0,0)^{T}\right\} \\
& =-\operatorname{sign}\left\{\sqrt{a_{1}^{2}-4 b_{1} h_{1}}\left[\left(\beta_{1} x_{+}-a_{2}+\sqrt{\left(\beta_{1} x_{+}-a_{2}\right)^{2}-4 b_{2} h_{2}}\right)^{2}-4 b_{2} h_{2}\right]\right. \\
& \left.\times\left[\left(\beta_{3} y_{-}^{+}-d_{1}-\sqrt{\left(\left(\beta_{3} y_{-}^{+}-d_{1}\right)^{2}-4 h_{3} a_{31}\right)}\right)^{2}-4 a_{31} h_{3}\right]\right\} \\
& =1 \text {, } \\
& \operatorname{deg}\left\{J Q N u, \Omega_{4} \cap \operatorname{Ker} L,(0,0,0)^{T}\right\} \\
& =-\operatorname{sign}\left\{\sqrt{a_{1}^{2}-4 b_{1} h_{1}}\left[\left(\beta_{1} x_{+}-a_{2}+\sqrt{\left(\beta_{1} x_{+}-a_{2}\right)^{2}-4 b_{2} h_{2}}\right)^{2}-4 b_{2} h_{2}\right]\right. \\
& \left.\times\left[\left(\beta_{3} y_{+}^{+}-d_{1}-\sqrt{\left(\left(\beta_{3} y_{+}^{+}-d_{1}\right)^{2}-4 h_{3} a_{31}\right)}\right)^{2}-4 a_{31} h_{3}\right]\right\} \\
& =1 \text {, }
\end{aligned}
$$




$$
\begin{aligned}
& \operatorname{deg}\left\{J Q N u, \Omega_{5} \cap \operatorname{Ker} L,(0,0,0)^{T}\right\} \\
& =-\operatorname{sign}\left\{-\sqrt{a_{1}^{2}-4 b_{1} h_{1}}\left[\left(\beta_{1} x_{-}-a_{2}-\sqrt{\left(\beta_{1} x_{-}-a_{2}\right)^{2}-4 b_{2} h_{2}}\right)^{2}-4 b_{2} h_{2}\right]\right. \\
& \left.\times\left[\left(\beta_{3} y_{-}^{-}-d_{1}+\sqrt{\left(\left(\beta_{3} y_{-}^{-}-d_{1}\right)^{2}-4 h_{3} a_{31}\right)}\right)^{2}-4 a_{31} h_{3}\right]\right\} \\
& =-1 \text {, } \\
& \operatorname{deg}\left\{J Q N u, \Omega_{6} \cap \operatorname{Ker} L,(0,0,0)^{T}\right\} \\
& =-\operatorname{sign}\left\{-\sqrt{a_{1}^{2}-4 b_{1} h_{1}}\left[\left(\beta_{1} x_{-}-a_{2}+\sqrt{\left(\beta_{1} x_{-}-a_{2}\right)^{2}-4 b_{2} h_{2}}\right)^{2}-4 b_{2} h_{2}\right]\right. \\
& \left.\times\left[\left(\beta_{3} y_{+}^{-}-d_{1}+\sqrt{\left(\left(\beta_{3} y_{+}^{-}-d_{1}\right)^{2}-4 h_{3} a_{31}\right)}\right)^{2}-4 a_{31} h_{3}\right]\right\} \\
& =1 \text {, } \\
& \operatorname{deg}\left\{J Q N u, \Omega_{7} \cap \operatorname{KerL},(0,0,0)^{T}\right\} \\
& =-\operatorname{sign}\left\{\sqrt{a_{1}^{2}-4 b_{1} h_{1}}\left[\left(\beta_{1} x_{+}-a_{2}+\sqrt{\left(\beta_{1} x_{+}-a_{2}\right)^{2}-4 b_{2} h_{2}}\right)^{2}-4 b_{2} h_{2}\right]\right. \\
& \left.\times\left[\left(\beta_{3} y_{-}^{+}-d_{1}+\sqrt{\left(\left(\beta_{3} y_{-}^{+}-d_{1}\right)^{2}-4 h_{3} a_{31}\right)}\right)^{2}-4 a_{31} h_{3}\right]\right\} \\
& =-1 \text {, } \\
& \operatorname{deg}\left\{J Q N u, \Omega_{8} \cap \operatorname{Ker} L,(0,0,0)^{T}\right\} \\
& =-\operatorname{sign}\left\{\sqrt{a_{1}^{2}-4 b_{1} h_{1}}\left[\left(\beta_{1} x_{+}-a_{2}+\sqrt{\left(\beta_{1} x_{+}-a_{2}\right)^{2}-4 b_{2} h_{2}}\right)^{2}-4 b_{2} h_{2}\right]\right. \\
& \left.\times\left[\left(\beta_{3} y_{+}^{+}-d_{1}+\sqrt{\left(\left(\beta_{3} y_{+}^{+}-d_{1}\right)^{2}-4 h_{3} a_{31}\right)}\right)^{2}-4 a_{31} h_{3}\right]\right\} \\
& =-1 \text {. }
\end{aligned}
$$

So far, we have proved that $\Omega_{i}(i=1, \ldots, 8)$ satisfies all the assumptions in Theorem 2.1. Hence, system (2.2) has at least eight different periodic solutions. By (2.1), system (1.2) has at least eight different positive $\omega$-periodic solutions. This completes the proof.

\section{Examples.}

EXAMPLE 1. Consider the following predator-prey system with stocking:

$$
\left\{\begin{array}{l}
\frac{d x_{1}}{d t}=x_{1}\left[a_{1}(t)-b_{1}(t) x_{1}(t)-c_{1}(t) x_{2}(t)\right]-h_{1}(t), \\
\frac{d x_{2}}{d t}=x_{2}\left[-a_{2}(t)-b_{2}(t) x_{2}(t)+\beta_{1}(t) x_{1}(t-\tau)-\beta_{2}(t) x_{3}(t)\right]-h_{2}(t), \\
\frac{d x_{3}}{d t}=x_{3}\left[-d_{1}(t)+\beta_{3}(t) x_{2}(t-\tau)-a_{31}(t) x_{3}(t)\right]-h_{3}(t),
\end{array}\right.
$$


where all coefficients are positive continuous $\omega$-periodic functions and $\tau>0$ is a constant delay.

In Theorem 2.2, $g_{1}\left(t, x_{1}\right)=a_{1}(t)-b_{1}(t) x_{1}(t), g_{2}\left(t, x_{2}\right)=a_{2}(t)+b_{2}(t) x_{2}(t), p_{2}(x)=$ $p_{3}(x)=x$. If $x \geq \ln \frac{a_{1}^{M}}{b_{1}^{L}} \stackrel{\text { def }}{=} A$, then $g_{1}\left(t, e^{x}\right) \leq 0$, and if $x \geq \ln \frac{\beta_{1}^{M} e^{A}}{b_{2}^{L}} \stackrel{\text { def }}{=} B$, then $g_{2}\left(t, e^{x}\right) \geq \beta_{1}^{M} p_{2}\left(e^{A}\right), t, x \in R$. Take

$$
\begin{gathered}
l_{ \pm}=\frac{1}{2 b_{1}^{M}}\left\{a_{1}^{l}-c_{1}^{M} e^{B} \pm \sqrt{\left(a_{1}^{l}-c_{1}^{M} e^{B}\right)^{2}-4 b_{1}^{M} h_{1}^{M}}\right\}, \\
u_{ \pm}=\frac{1}{2 b_{2}^{M}}\left\{\frac{\beta_{1}^{l} h_{1}^{l}}{a_{1}^{M}}-a_{2}^{M}-\frac{\beta_{2}^{M} \beta_{3}^{M} e^{B}}{a_{31}^{l}} \pm \sqrt{\left(\frac{\beta_{1}^{l} h_{1}^{l}}{a_{1}^{M}}-a_{2}^{M}-\frac{\beta_{2}^{M} \beta_{3}^{M} e^{B}}{a_{31}^{l}}\right)^{2}-4 b_{2}^{M} h_{2}^{M}}\right\},
\end{gathered}
$$

then when $\ln l_{-} \leq x \leq \ln l_{+}$,

$$
e^{x} g_{1}\left(t, e^{x}\right) \geq c_{1}^{M} e^{B} p_{2}\left(e^{x}\right)+h_{1}^{M},
$$

and when $\ln u_{-} \leq x \leq \ln u_{+}$,

$$
h_{2}^{M}+\frac{\beta_{2}^{M} \beta_{3}^{M}}{a_{31}^{l}} p_{3}\left(e^{B}\right) p_{3}\left(e^{x}\right)+e^{x} g_{2}\left(t, e^{x}\right) \leq \beta_{1}^{l} e^{x} p_{2}\left(\frac{h_{1}^{l}}{a_{1}^{M}}\right) .
$$

Hence if the following conditions hold:

(H1) $a_{1}^{l}>c_{1}^{M} e^{B}+2 \sqrt{b_{1}^{M} h_{1}^{M}}$;

(H2) $\frac{\beta_{1}^{l} h_{1}^{l}}{a_{1}^{M}}>a_{2}^{M}+\frac{\beta_{2}^{M} \beta_{3}^{M} e^{B}}{a_{31}^{l}}+2 \sqrt{b_{2}^{M} h_{2}^{M}}$;

(H3) $\frac{\beta_{3}^{l} h_{2}^{l}}{\beta_{1}^{M} e^{A}}>d_{1}^{M}+2 \sqrt{a_{31}^{M} h_{3}^{M}}$,

then all conditions in Theorem 2.2 are satisfied. Therefore, by Theorem 2.2, we have the following theorem.

Theorem 3.1. Assume that (H1)-(H3) hold. Then system (3.1) has at least eight positive $\omega$-periodic solutions.

EXAMPLE 2. Consider the following predator-prey system with functional response and stocking given by

$$
\left\{\begin{aligned}
\frac{d x_{1}}{d t} & =x_{1}\left(a_{1}(t)-b_{1}(t) x_{1}(t)-\frac{c_{1}(t) x_{2}(t)}{m x_{1}(t)+\gamma}\right)-h_{1}(t) \\
\frac{d x_{2}}{d t} & =x_{2}\left(-a_{2}(t)-b_{2}(t) x_{2}(t)+\frac{\beta_{1}(t) x_{1}(t-\tau)}{m x_{1}(t-\tau)+\gamma}-\frac{\beta_{2}(t) x_{3}(t)}{n x_{2}(t)+p}\right)-h_{2}(t), \\
\frac{d x_{3}}{d t} & =x_{3}\left(-d_{1}(t)+\frac{\beta_{3}(t) x_{2}(t-\tau)}{n x_{2}(t-\tau)+p}-a_{31}(t) x_{3}(t)\right)-h_{3}(t)
\end{aligned}\right.
$$

where all coefficients are positive continuous $\omega$-periodic functions, and $x>0$ is a constant delay.

In Theorem 2.2, $g_{1}(t, x)=a_{1}(t)-b_{1}(t) x_{1}(t), g_{2}(t, x)=a_{2}(t)+b_{2}(t) x_{2}(t), p_{2}(x)=$ $\frac{x_{1}(t)}{m(t) x_{1}(t)+r(t)}, p_{3}(t)=\frac{x_{2}(t)}{n(t) x_{2}(t)+p(t)}$. If $x \geq \ln \frac{a_{1}^{M}}{b_{1}^{l}} \stackrel{\text { def }}{=} A$, then $g_{2}\left(t, e^{x}\right) \leq 0, t, x \in R$ and 
if $x \geq \ln \frac{\beta_{1}^{M} e^{A}}{b_{2}^{l}} \stackrel{\text { def }}{=} B$, then $g_{2}\left(t, e^{x}\right) \geq \beta_{1}^{M} p_{2}\left(e^{A}\right), t, x \in R$. Let

$$
\begin{aligned}
l_{ \pm} & =\frac{1}{2 b_{1}^{M}}\left\{a_{1}^{l}-\frac{c_{1}^{M} e^{B}}{r^{l}} \pm \sqrt{\left.\left(a_{1}^{l}-\frac{c_{1}^{M} e^{B}}{r^{l}}\right)^{2}-4 b_{1}^{M} h_{1}^{M}\right\}},\right. \\
u_{ \pm} & =\frac{1}{2 b_{2}^{M}}\left\{\frac{\beta_{1}^{l} h_{1}^{l}}{a_{1}^{M} r^{M}+m^{M} h_{1}^{l}}-a_{2}^{M}-\frac{\beta_{2}^{M} \beta_{3}^{M}}{a_{31}^{l} n^{l} p^{l}}\right. \\
& \left. \pm \sqrt{\left(\frac{\beta_{1}^{l} h_{1}^{l}}{a_{1}^{M} r^{M}+m^{M} h_{1}^{l}}-a_{2}^{M}-\frac{\beta_{2}^{M} \beta_{3}^{M}}{a_{31}^{l} n^{l} p^{l}}\right)^{2}}-4 b_{2}^{M} h_{2}^{M}\right\} .
\end{aligned}
$$

Then if $\ln l_{-} \leq x \leq \ln l_{+}, e^{x} g_{1}\left(t, e^{x}\right) \geq c_{1}^{M} e^{B} p_{2}\left(e^{x}\right)+h_{1}^{M}$ and if $\ln u_{-} \leq x \leq \ln u_{+}$, then $h_{2}^{M}+\frac{\beta_{2}^{M} \beta_{3}^{M}}{a_{31}^{l}} p_{3}\left(e^{B}\right) p_{3}\left(e^{x}\right)+e^{x} g_{2}\left(t, e^{x}\right) \leq \beta_{1}^{l} e^{x} p_{2}\left(\frac{h_{1}^{l}}{a_{1}^{M}}\right), t, x \in R$. Hence if the following conditions hold,

(T1) $a_{1}^{l}>\frac{c_{1}^{M} e^{B}}{r^{l}}+2 \sqrt{b_{1}^{M} h_{1}^{M}}$;

(T2) $\frac{\beta_{1}^{l} h_{1}^{l}}{a_{1}^{M} r^{M}+m^{M} h_{1}^{l}}>a_{2}^{M}+\frac{\beta_{2}^{M} \beta_{3}^{M}}{a_{31}^{l} n^{l} p^{l}}+2 \sqrt{b_{2}^{M} h_{2}^{M}}$;

(T3) $\beta_{3}^{l} h_{2}^{l}>\left(n^{M} h_{2}^{l}+\frac{p^{M} e^{A} \beta_{1}^{M}}{m^{M} e^{A}+r^{M}}\right)\left(d_{1}^{M}+2 \sqrt{a_{31}^{M} h_{3}^{M}}\right)$,

then all requirements in Theorem 2.2 are satisfied. Therefore, by Theorem 2.2, we obtain the following theorem.

Theorem 3.2. Assume that (T1)-(T3) hold. Then system (3.2) has at least eight different positive $\omega$-periodic solutions.

EXAMPLE 3. Consider the system

$$
\left\{\begin{array}{r}
\frac{d x_{1}}{d t}=x_{1}\left[99+\sin t-(101+\sin t) x_{1}(t)-\frac{1}{100+\sin t} x_{2}(t)\right]-21-\sin t \\
\frac{d x_{2}}{d t}=x_{2}\left[-\frac{1}{100+\cos t}-(101+\cos t) x_{2}(t)\right. \\
\left.+(99+\sin t) x_{1}(t-\tau)-(9+\sin t) x_{3}(t)\right]-\frac{1}{9+\cos t} \\
\frac{d x_{3}}{d t}=x_{3}\left[-\frac{1}{11+\cos t}+(999+\cos t) x_{2}(t-\tau)\right. \\
\left.-(10001+\sin t) x_{3}(t)\right]-\frac{1}{90001+\sin t} .
\end{array}\right.
$$

It is easy to examine that the coefficients of system (3.3) satisfy all assumptions in Theorem 3.1. Thus, by Theorem 3.1, system (3.3) has at least eight positive $\omega$-periodic solutions.

\section{REFERENCES}

[1] L. Chen and J. Chen, Nonlinear Dynamical System in Biology (Science Press, Beijing, 1993).

[2] M. Farkas and H. J. Freedman, The stable coexistence of competing species an an renewable resource, J. Math. Anal. Appl. 138(1989), 401-472.

[3] H. I. Freedman and S. Ruan, Uniform persistence in functional differential equations, J. Differential. Equations 115(1995), 173-192. MR1308612 (96e:34115) 
[4] H. I. Freedman and P. Waltman, Persistence in three interacting predator-prey populations, Math. Biosci. 88(1984), 213-231. MR.738903 (85h:92037)

[5] R. E. Gaines and J. L. Mawhin, Coincidence Degree and Nonlinear Differetial Equation (SpringerVerlag, 1977). MR0637067 (58:30551)

[6] J. L. Mawhin, Topological Degree Methods in Nonlinear Boundary Value problems. CBMS Reg. Conf. Ser. Math. 40 (Amer, Math. Soc., Providence, 1979). MR.525202 (80c:47055)

[7] D. Mukherjee and A. B. Roy, Uniform persistence and global attractivity theorem for generalized prey-predator system with time delay, J. Nonlin Anal. TMA 38 (1999), 59-74. MR1693012 (2000e:92031)

[8] Z. Q. Zhang, Z. C. Wang, The existence of a periodic solution for a generalized prey-predator system with delay, Math. Proc Camb Phil. Soc. 137 (2004), 475. MR2092072 (2005e:34221) 